\title{
Integrating Wireless Systems into Process Industry and Business Management
}

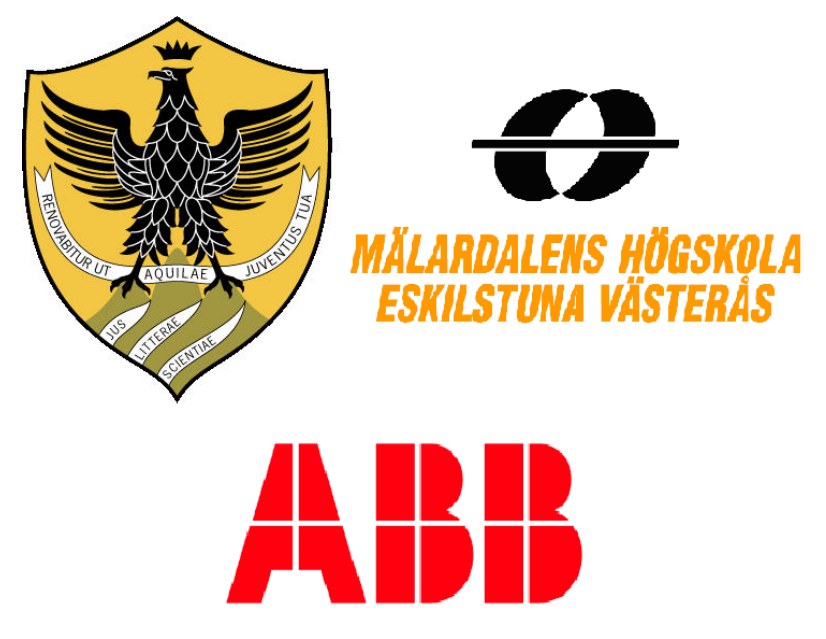

Federico Ciccozzi

Department of Computer Science

Universitá degli Studi di L'Aquila, Italy

Mälardalens Högskola, Västerås, Sweden

ABB Corporate Research, Västerås, Sweden

A thesis submitted for the degree of Master of Science in Global Software Engineering September 2009 
I would like to dedicate this thesis to my beloved friends Alessandra, Giovanni and Lorenzo who have been taken away from their young lives by the earthquake in L'Aquila on the 6th April 2009, and will live forever in my heart. 


\section{Acknowledgements}

And I would like to acknowledge all who played a role in my project either directly or indirectly. I begin by thanking my family, who always supported me and my decisions and allowed me to concetrate on my studies without any worry. Then, I would like to thank my supervisor at ABB Corporate Research, Tiberiu Seceleanu, who has always taken time from his tight schedule to give me all the help I needed in every phase of the project. The insights provided by both him and Johan Akerberg expanded my knowledge and problems understanding, enriching the results profoundly. I would like to thank Prof. Ivica Crnković, whose helpfulness facilitates the whole thesis process, from the first application details to the very final steps. I also extend my gratitude to my supervisor at MDH, Antonio Cicchetti, who was always ready to listen to my problems and give me very useful hints, even when he was on vacation. I especially thank Prof. Henry Muccini from Universitá degli Studi di L'Aquila, since without his devotion to the GSEEM program I could not have had the possibility to live this exciting and important experience abroad. A final special thanks to all my friends from all over the world who filled and fill my life with a countless number of wonderful moments.

At the very end I thank myself... 


\begin{abstract}
We analyze here the topic of integration, in the area of factory automation, from sensor/actuator levels to plant management levels. The communication at the plant level is based on wireless technology while management applications run in supervisory concentrators but can also be distributed, communicating via the Internet. The work reported here is part of SOCRADES, a European advanced research and development project aiming to build a design, execution and management platform for next-generation industrial automation systems. While theoretical and simulation level results have been previously reached, our final goal here is a real-life demonstrator at a mining plant - New Boliden (Boliden, Sweden). The thesis work is a continuation of the previous work in the project, and provides support for the integration of WirelessHART networks at the plant level, and a management application dialogue between ABB and SAP systems, two main partners in the SOCRADES project. A small control environment is to be deployed at the Boliden plant, to supervise a tank level control system. The plant is connected, via ABB control and management applications and servers to high management procedures of the partners. Targeted results of the MSc work are an interface between wireless and wired systems, the deployment of a control environment at Boliden, the development of the enterprise business management facilities and the realization of a software package to support the WirelessHART solution.
\end{abstract}




\section{Contents}

Nomenclature viii

1 Introduction 1

1.1 Thesis Topic . . . . . . . . . . . . . . . . 3

1.2 Outline of the thesis . . . . . . . . . . . 5

1.2.1 Chapter 2 - Wired Networks . . . . . . . . . . 5

1.2.2 Chapter 3 - Wireless Networks . . . . . . . . . . 5

1.2.3 Chapter 4 - Involved ABB's Industrial IT Devices . . . . . 6

1.2.4 Chapter 5 - Integrating the Plant . . . . . . . . . . . . 6

1.2.5 Chapter 6 - Integrating the Management . . . . . . . . . 6

$\begin{array}{llr}2 & \text { Wired Networks } & 7\end{array}$

2.1 Fieldbus Foundation . . . . . . . . . . . . . . . . 8

2.2 PROFINET . . . . . . . . . . . . . . . . . . . 9

2.3 MODBUS . . . . . . . . . . . . . . . . . 10

2.3.1 Protocol description . . . . . . . . . . . . . 13

2.3.2 Data model . . . . . . . . . . . . . . . . . 15

2.3.3 A MODBUS library in C: libmodbus . . . . . . . . . 16

$\begin{array}{llr}3 & \text { Wireless Networks } & 17\end{array}$

3.1 Wireless Communication . . . . . . . . . . . . . . . 17

3.2 Wireless Common Protocols . . . . . . . . . . . . . . 17

3.2 .1 The IEEE $802 \ldots \ldots \ldots \ldots \ldots$

3.2.1.1 The IEEE $802.11 \ldots \ldots \ldots$. . . . . . . . . 18

3.2.1.2 The IEEE $802.15 \ldots \ldots \ldots$ 
3.2 .2 Bluetooth . . . . . . . . . . . . . . . . . . . 19

3.2 .3 ZigBee . . . . . . . . . . . . . . . . . . 20

3.2 .4 WiMax . . . . . . . . . . . . . . . . . . 20

3.3 WirelessHART . . . . . . . . . . . . . . . 20

3.3.1 The WirelessHART Architecture . . . . . . . . . . . 21

3.3.2 The WirelessHART Network . . . . . . . . . . . . . . . 23

3.3.3 The Dust Network Manager . . . . . . . . . . . . . . . 24

3.3.3.1 XML-RPC Communication Protocol . . . . . . 25

3.3.3.2 XML-RPC Protocol Description . . . . . . . . . 25

3.3.3.3 A XML-RPC library in C: xmlrpc-c . . . . . . 29

4 Involved ABB's Industrial IT Devices 31

4.1 ABB's Industrial IT 800xA System . . . . . . . . . . . . . 31

4.2 ABB's Industrial IT cpmPlus Enterprise Connectivity . . . . . . . 33

5 Integrating the Plant 36

5.1 Development Process of the Communication Manager Application 37

5.2 Requirements Specification . . . . . . . . . . . . . . 38

5.2 .1 Functional Requirements . . . . . . . . . . . . . . . . 38

5.2 .2 Extra-Functional Requirements . . . . . . . . . . . . . 41

5.3 Design Specification . . . . . . . . . . . . . . . . . 42

5.3 .1 Conceptual Design . . . . . . . . . . . . . . . . . 42

$5.3 .2 \quad$ Structural Design . . . . . . . . . . . . . . . . 43

5.3 .3 Behavioral Design . . . . . . . . . . . . . . . . . 45

5.4 Implementation . . . . . . . . . . . . . . . 55

5.4 .1 Development Environment Preparation . . . . . . . . . 55

5.4 .2 Connections Handler . . . . . . . . . . . . . . . 57

5.4 .3 MODBUS Slave Thread . . . . . . . . . . . . 58

5.4 .4 Dust Client Thread . . . . . . . . . . . . . . . . . . . . 58

5.4.5 Shared Memory Handler . . . . . . . . . . . . . . . . . 61

5.4 .6 XML Manager . . . . . . . . . . . . . . . . . . . . 62

5.4.7 Shared Memory Composition . . . . . . . . . . . . 62 
$\begin{array}{lll}6 & \text { Integrating the Management } & 64\end{array}$

6.1 Plant Operator's Perspective . . . . . . . . . . . . . . 65

6.2 Enterprise Business Management's Perspective . . . . . . . . . 67

$\begin{array}{lll}7 & \text { Conclusions } & 74\end{array}$ 


\section{List of Figures}

1.1 The SOCRADES consortium . . . . . . . . . . . . 2

1.2 A view of the ideal solution . . . . . . . . . . . . . 3

1.3 A view of the actual solution for the live demonstration . . . . . . 4

1.4 The future plant with WirelessHART control . . . . . . . . . . 5

2.1 The OSI Layered model _ . . . . . . . . . . . . . . . . . . 11

2.2 An example of a MODBUS network architecture . . . . . . . . . . 12

2.3 MODBUS frame composition . . . . . . . . . . . . . . 14

3.1 A WirelessHART Network . . . . . . . . . . . . . . . . 22

3.2 WirelessHART Network Topologies . . . . . . . . . . . . . . 23

3.3 XML-RPC Request Example . . . . . . . . . . . . . . . . . . 26

3.4 XML-RPC Response Example . . . . . . . . . . . . . . . . . 26

3.5 XML-RPC Array Type . . . . . . . . . . . . . . . . . . . . . . 28

3.6 XML-RPC Multidimensional Array Type . . . . . . . . . . . . . 28

3.7 XML-RPC Struct Type . . . . . . . . . . . . . . . . . . . . . . . . 29

4.1 A physical view of the 800xA System . . . . . . . . . . . 32

4.2 ECS Web Service Connectivity . . . . . . . . . . . . . 34

5.1 Development process V-model . . . . . . . . . . . . . . . 37

5.2 Conceptual Model . . . . . . . . . . . . . . . . . . . . . . . 42

5.3 Structural model in UML . . . . . . . . . . . . . . . . . . 44

5.4 Start application sequence diagram . . . . . . . . . . . . . . 46

5.5 MODBUS slave initialization sequence diagram . . . . . . . . . 47

5.6 MODBUS read request handling sequence diagram . . . . . . . . 48 
5.7 MODBUS write request handling sequence diagram . . . . . . . . 49

5.8 Dust client initialization sequence diagram . . . . . . . . . . 50

5.9 Dust read/write request sending sequence diagram . . . . . . . . 51

5.10 Dust notifications handling sequence diagram . . . . . . . . . 53

5.11 Polling Devices Information Requests sequence diagram . . . . . . 54

6.1 Boliden Operator Workplace's view . . . . . . . . . . . . . 65

6.2 ECS Complete Model . . . . . . . . . . . . . . . . . . . . . . 68

6.3 ECS Web Service Integration . . . . . . . . . . . . . . . 69

6.4 ReadProperty Request . . . . . . . . . . . . . . . . . 71

6.5 ReadProperty Response . . . . . . . . . . . . . . . . 71

6.6 ExecutionEvent Request . . . . . . . . . . . . . . . 73 


\section{Chapter 1}

\section{Introduction}

Wireless technology is getting more and more widespread due to its several advantages: no more need for expensive and 'limited' cables, possibility to connect to areas unreachable through cables and so on. From an industrial point of view, the migration towards the wireless technology was due to the ease of deployment and reconfiguration of wireless connections together with the elimination of installation and maintenance of standard cabling, which reduce both cost and time. Nevertheless, its lack of maturity and the consequent difficulties in providing realtime performance and reliability metrics in comparison to wired networks, do not help the application of the wireless technology in industrial environments.

This thesis work is part of an European advanced research and development project (fig. 1.1), called Service Oriented Cross-layer infRAstructure for Distributed smart Embedded deviceS (SOCRADES), and carried out by a cooperation between several top companies (ABB, APS, ARM, Boliden, FlexLink, Ifak, Jaguar Cars Ltd., SAP, Schneider Electric, Siemens) and universities (KTH, Loughborough University, LTU, Politecnico di Milano, TUT). The goal of the SOCRADES project [1] is to develop a design, execution and management platform for next-generation industrial automation systems; the main objective is the specification of a new wireless communication architecture that provides the required reliability, safety, security and real-time parameters for wireless sensor networks. Algorithms and software packages have been previously developed for the realization of a real-life demonstrator at the Boliden Mining Plant. This thesis work provides an interface which allows the exchange of information among 
devices which use different communication protocols.

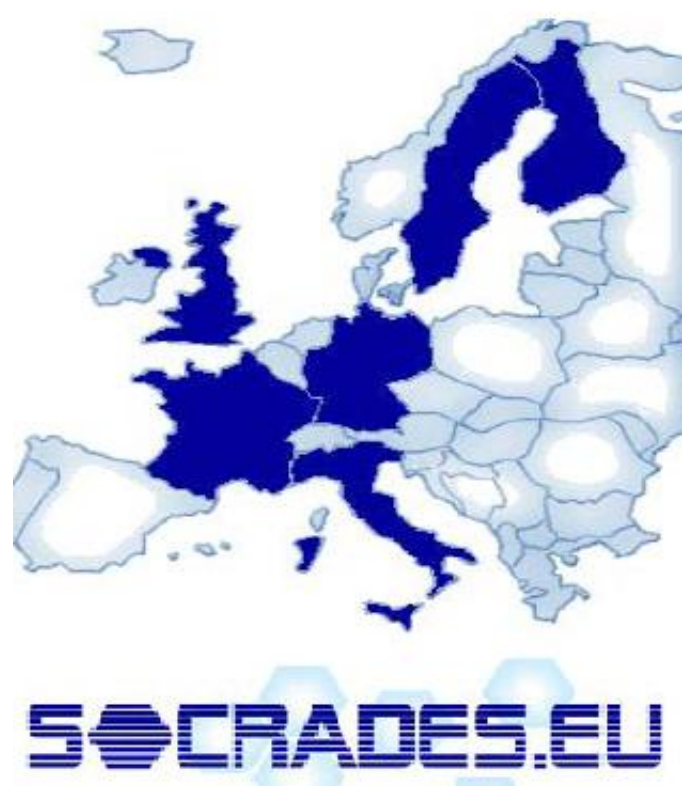

Figure 1.1: The SOCRADES consortium 


\subsection{Thesis Topic}

\section{$1.1 \quad$ Thesis Topic}

The final product of this thesis work is a system whose main component is a communication manager application, which handles the exchange of information between a wireless network of sensors placed at the Boliden Mining Plant and a controller system (ABB's 800xA). Moreover the system will be able to forward information about the network to the enterprise business layer through the ABB's cpmPlus Enterprise Connectivity (ECS) Framework. Sensors at Boliden will be connected to a wireless network that will have to communicate with the 800xA Controller System [2] even though they use different communication protocols. In the ideal solution (fig. 1.2), with WirelessHART as wireless protocol, this gap will be filled by the communication manager application which will act as a bridge between the XML-RPC/XML communication protocol used by the ELPRO 245U-E WirelessHART Gateway, which includes a Dust Network Manager and a WirelessHART Gateway [3], and the MODBUS/TCP communication protocol used by the 800xA Controller System. The system will reach full completeness by the ability of forwarding information about the network from the 800xA System to the enterprise business layer. This operation will be carried out through the connectivity provided by the ABB's ECS framework 4.0.

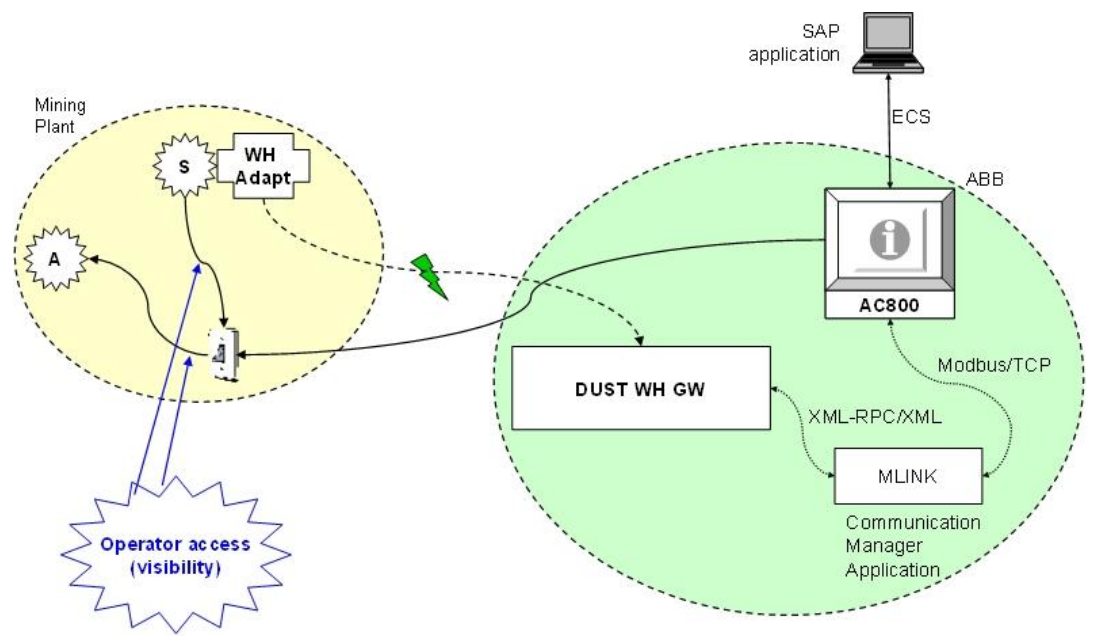

Figure 1.2: A view of the ideal solution 


\subsection{Thesis Topic}

Due to the unavailability of WirelessHART devices for the final love demonstration of the SOCRADES project, has selected Zigbee as wireless protocol. This means that the communication manager application described in this report will not be used for it, but rather in further developments in which WirelessHART devices will be available. The figure 1.3 shows a view of the final live demonstration system.

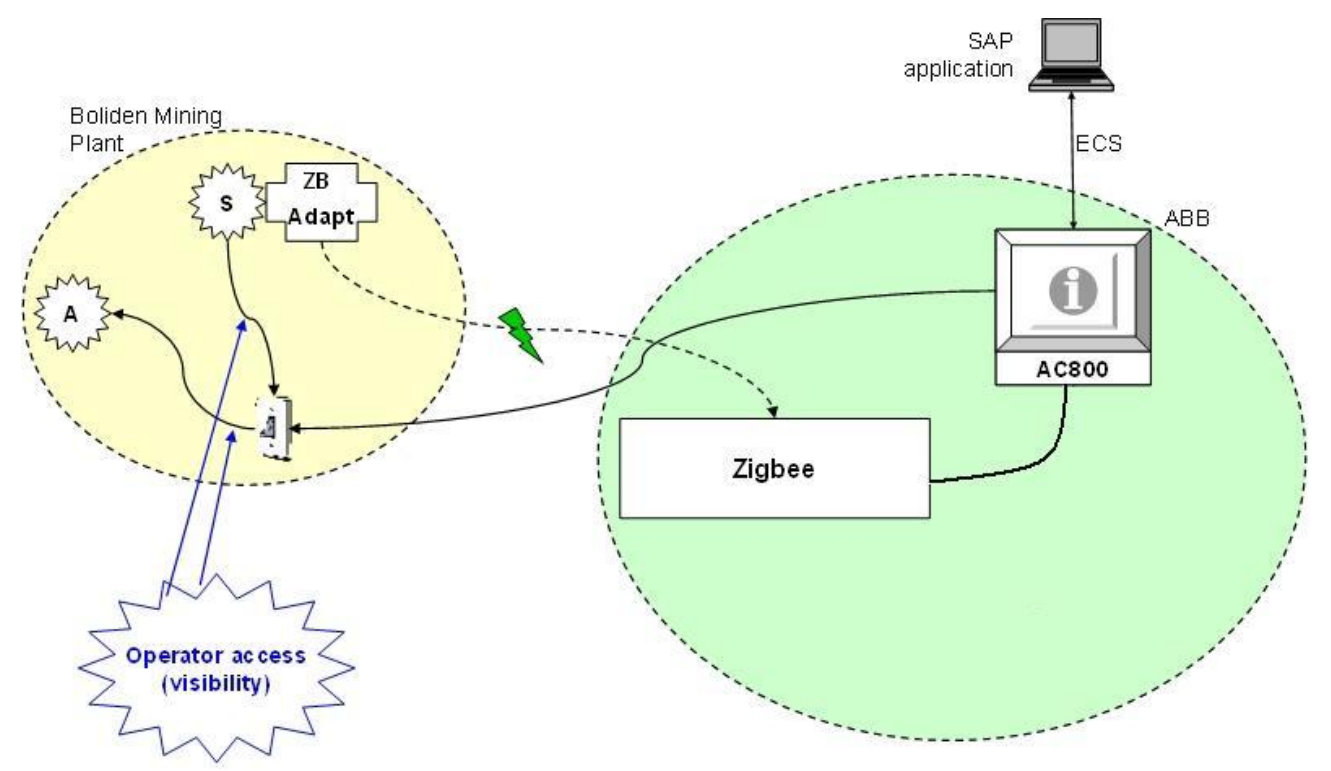

Figure 1.3: A view of the actual solution for the live demonstration

The future goal is to apply the same integration process using WirelessHART as wireless protocol, since it is becoming the de-facto standard protocol for wireless systems in process industry mostly because, as a standards-based technology, it ensures multi-vendor interoperability of a multitude of field devices in process operations. In the figure 1.4, a future plant with WirelessHART control is shown.

Details of each part of the final product together with a description of the development process will be given in the following sections. 


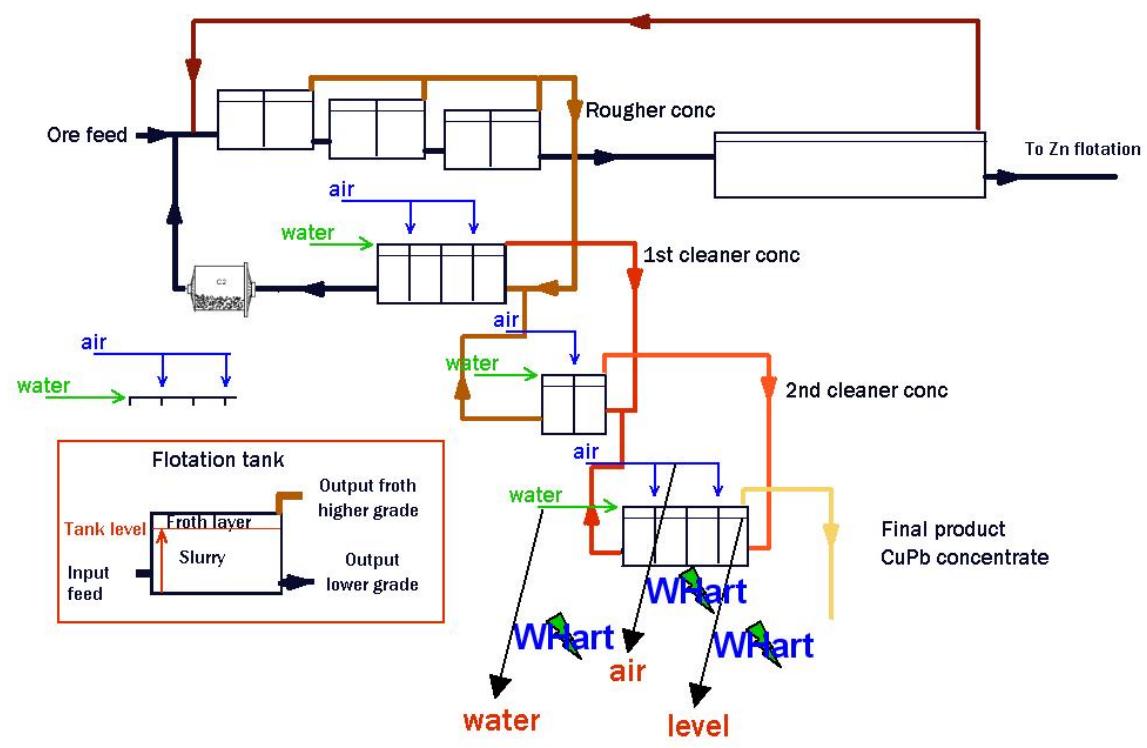

Figure 1.4: The future plant with WirelessHART control

\subsection{Outline of the thesis}

This thesis report is composed by the following sections:

\subsubsection{Chapter 2 - Wired Networks}

This work involves not only wireless communication. In fact it provides a sort of bridge connection between a wireless system (WirelessHART network) and a wired system (Controller device via MODBUS/TCP). In this section an overview of wired networks and protocols will be given, paying particular attention to the MODBUS protocol.

\subsubsection{Chapter 3 - Wireless Networks}

In this section an overview of wireless networks and the most wide-spread wireless protocols will be given, with special focus on the WirelessHART technology, which represents a central issue for the thesis work. 


\subsubsection{Chapter 4 - Involved ABB's Industrial IT Devices}

The 800xA System is a primary component of the final system. This section aims to give an overview on this component in order to facilitate the understanding of its role in the process.

The final system will have to be able to facilitate the business management by providing communication between the control system itself and the business management systems through the BB's Industrial IT cpmPlus Enterprise Connectivity (ECS). In this section an overview on the ECS framework will be given, focusing on the features that have been used for our purposes.

\subsubsection{Chapter 5 - Integrating the Plant}

In this section the entire development process of the final product will be described in details. Starting from the specification of functional and extra-functional requirements and the tools selection, we will go through the actual development of the communication manager application. Explanation on how the application is intended to fulfil the specified requirements will be also given.

\subsubsection{Chapter 6 - Integrating the Management}

The final live demonstration scenario will be presented from the point of view of both the plant operator (through the ABB's 800xA System) and the enterprise business manager (through the ECS Web Service Integration). 


\section{Chapter 2}

\section{Wired Networks}

Before the advent of the wireless technology, computer networks [4] for industrial automation were built exclusively by using wired technologies. Fieldbus systems represent the most important communication systems used in commercial control installations. At the same time Ethernet, developed by Xerox PARC in 1975, represents a family of the most widespread computer networking technologies for Local Area Networks (LANs) within the office domain, standardized as IEEE 802.3 [5]. Due to its fast spread and consequent components low cost, in the last years there has been a noteworthy economic interest to widely introduce the Ethernet in computer networks for industrial automation. The most known industrial computer network protocols are:

- Fieldbus Foundation - it is a family of industrial computer network protocols particularly suited for real-time distributed control systems. It is widely used to establish connections between instruments in manufactory plants $[6]$.

- PROFINET - it is an open standard for industrial Ethernet mostly used for industrial automation applications [7].

- $M O D B U S$ - it is a family of industrial multidrop network based communication protocol to be used over a master/slave communication architecture [8]. 
- Ethernet Industrial Protocol (EtherNet/IP) - it is an open industrial communication protocol designed for industrial process control and other automation applications [9].

- DeviceNet - it is an industrial communication protocol developed for the interconnection of control devices in industrial automation applications [10].

- CANopen - it is a communication protocol for embedded systems in industrial automation environments. It provides different kinds of communication: master/slave, producer/consumer and client/server [11].

In the next sections a description of these three industrial computer network protocols will be given, focusing particularly on the MODBUS, which will be used for our purposes.

\subsection{Fieldbus Foundation}

Fieldbus Foundation represents a family protocols particularly suited for realtime distributed control in industrial networks. An example of its use might be the connection of instruments in a manufacturing plant. Fieldbus Foundation is not chained to any particular network structure since it can operate on star, ring, branch, tree and daisy-chain topologies. Several versions of Fieldbus Foundation have been released, but in this section we will focus on the Fieldbus Foundation H1. It makes use of both twisted pair and fiber media to allow the communication between multiple devices and the controller, which can communicate with upto 32 devices just by having a single communication point. This is one of the best improvements over the standard 4-20 mA communication that needed a communication point for each device. It supports several communication methods such as client/server, event based, scheduled/unscheduled, publisher/subscriber. Besides the improvements, Fieldbus Foundation has disadvantages compared to the older 4-20 mA analog signal standard: complexity, cost, speed and devices compatibility. 


\subsection{PROFINET}

PROFINET is an open standard for industrial Ethernet developed by PROFIBUSPROFINET International for automation. The standard is composed by two different perspectives:

- PROFINET CBA - it is particularly suitable for both component-based communication via TCP/IP and real-time communication in modular systems. The PROFINET CBA idea is that a whole automation system can be almost always divided into smaller autonomous sub-systems.

- PROFINET IO - it implements the interfacing with the peripherals by defining the entire data exchange between controllers, which act as masters, and devices, which can be seen as slaves. It follows the provider-consumer model and represents a good solution for fast data exchange between field devices over a WLAN. A typical PROFINET IO system is composed by three devices:

- IO Controller: it is responsible for all the control issues regarding the system

- IO Device: it is a field device that operates under the control of the Controller

- IO Supervisor: it is responsible for setting parameters and diagnosing IO Devices

In PROFINET IO systems, both data and alarms are trasmitted always in a real-time fashion.

PROFINET CBA and IO can communicate on the same bus at the same time and they can operate either separately or combined. The main advantages of PROFINET is its continuous further development that gives the users a longterm perspective for their PROFINET implementations, and the high degree of availability given by the PROFINET CBA autonomous subsystems. 


\section{$2.3 \quad$ MODBUS}

The Open Systems Interconnection reference model, well-known as OSI model, is an abstraction for layered communication. It basically divides the network architecture into seven different layers (fig. 2.1), numbered from top to bottom:

- 7 - Application Layer: it handles all the application management tasks by providing network services to the final user and defining interfaces used by the user processes for communicating in the network. Here is where the MODBUS communication protocol is placed.

- 6 - Presentation Layer: it provides data conversion from the user's representation to the canonical and vice versa.

- 5 - Session Layer: it drives the formatting of data to be exchanged over the connection.

- 4 - Transport Layer: it is the last abstract layer that acts as interface between the higher levels and the last three representing the physical network.

- 3 - Network Layer: it is responsible for all the issues regarding the actual communication from source to destination. It handles any problem related to the interconnection of heterogeneous networks.

- 2 - Data Link Layer: it checks the correctness of a bit sequence exchange between two nodes and requests for retransmission in case of transmission corruptions.

- 1 - Physical Layer: it handles all the details about the physical connection between the nodes.

A layer can be viewed as a set of conceptually similar functions providing a number of services to the layer above it and receiving other services from the layer below it. MODBUS is a messaging protocol positioned at level 7 of the OSI model, the application layer. Its main goal is to allow client/server communication between devices which use different types of buses or that are connected to different networks. It was developed in the 1979 by PLC manufacturer Modicon, 


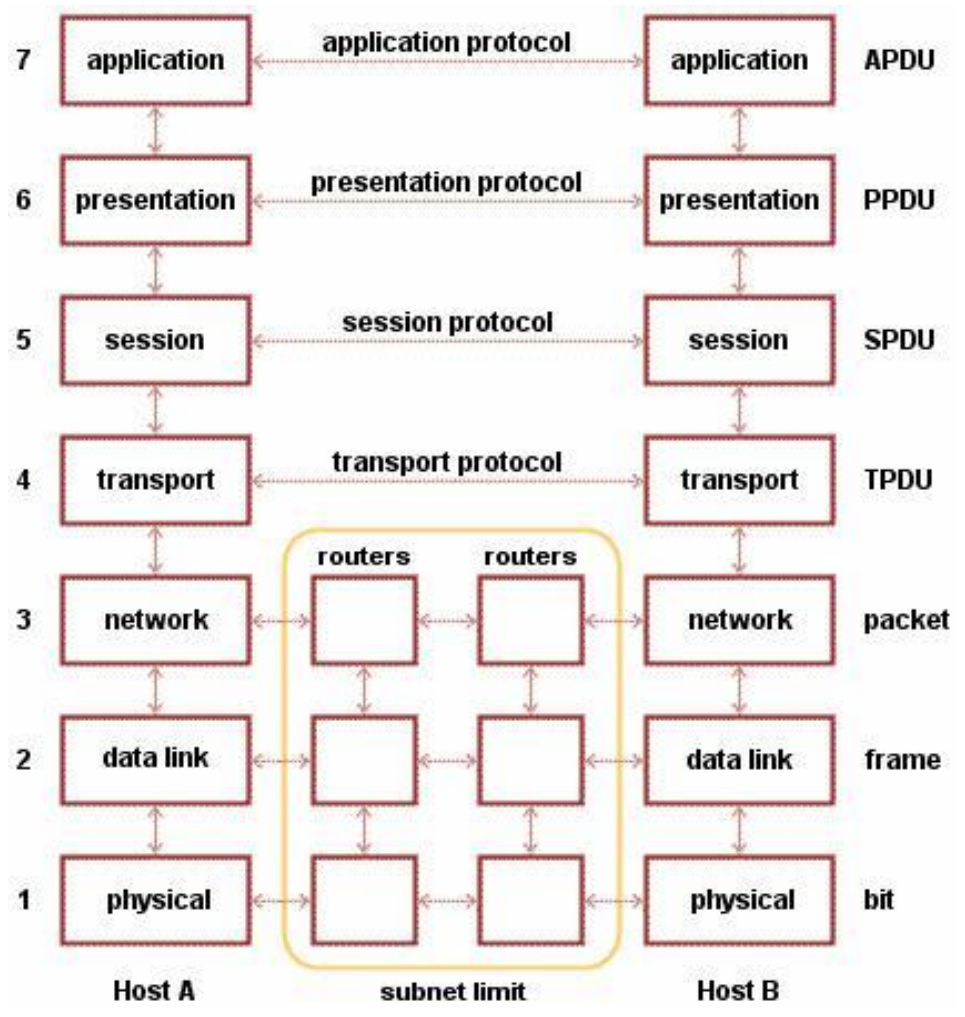

Figure 2.1: The OSI Layered model 
now part of Schneider Electric's Telemecanique. The MODBUS communication interface released by Modicon was intended as a multidrop network based communication over a master/slave architecture (fig. 2.2). The actual communication between MODBUS devices was achieved by using messages whose structure was released as an open standard. The first versions of the MODBUS interface ran on the RS-232 serial communication interface for computers and devices. Due to the needs of longer distances and higher speeds of communication, the RS-232 was later replaced by the RS-485, which also provided the possibility of a real multi-drop network.

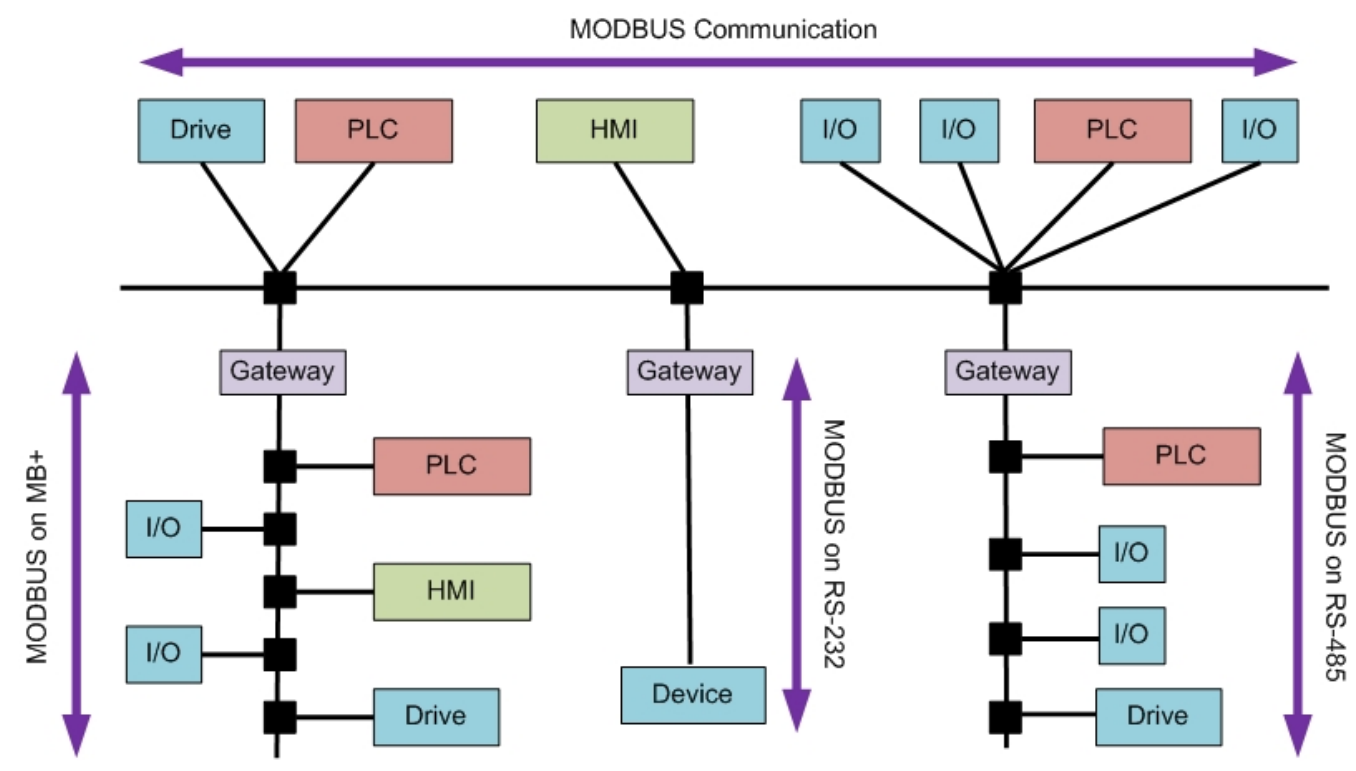

Figure 2.2: An example of a MODBUS network architecture

The MODBUS protocol soon became a de-facto standard for industrial networks thanks to its goodness, flexibility and simplicity. These characteristics encouraged a huge number of vendors to implement the MODBUS messaging system on their devices. Initially, MODBUS was mainly intended for wired serial communication liens, but it has evolved and been extended in order to support wireless communication and TCP/IP networks, which has been used in this project. 


\subsubsection{Protocol description}

The MODBUS communication interface is based on messages whose format does not depend on the chosen physical interface. A MODBUS device can communicate with several devices at once, even if their interfaces differ from each other. On simple serial communication physical interfaces like RS-232 and RS-485, the messages are exchanged in a plain form over the network that is dedicated to the MODBUS communication. In the case that the communication involves more complex network systems, such as TCP/IP over Ethernet, the messages are embedded in packets following the format requested by the physical interface. This is the case in which MODBUS and other different connections can share the same physical interface, which is no more dedicated to the single MODBUS. Embedded or plain, the MODBUS messages have always the same structure which is composed by four basic elements:

- Device address - It specifies the address of the receiver

- Function code - It defines the message type:

- 01 - Read coil status: a coil is a discrete output value and this function can be used to read the status of such an output

- 02 - Read input status: input values are read in the same way as the status of coils, but using this function instead

- 03 - Read holding registers: holding registers are used to store internal values of a device. This function allows to read one or more holding register values from a specified slave

- 04 - Read input registers: input registers are read-only values which can be read through this function

- 05 - Force single coil: a coil value can be either read or written. This function is used to force the value of a single coil

- 06 - Preset single register: an holding register value can be either read or written. This function is used to write the value of an holding register 
- 07 - Read exception status: it can be used to request the exception status of a slave device

- 15 - Force multiple coils: as the function 05, it forces coil values, but it's used to operate on multiple coils at the same time

- 16 - Preset multiple registers: as the function 06, it presets holding register values, but it's used to operate on multiple holding registers at the same time

- 17 - Report slave ID: it is used to retrieve the ID of a selected slave device

- Data - It represents a data block with some additional information

- Error check - It is a CRC-32 error check code which results in an infinitesimal chance of undetected corruption to a request or response message

Function Code and Data compose the Protocol Data Unit (PDU, fig. 2.3), that together with the Device Address and the Error Check compose the Application Data Unit (ADU, fig. 2.3).

ADU

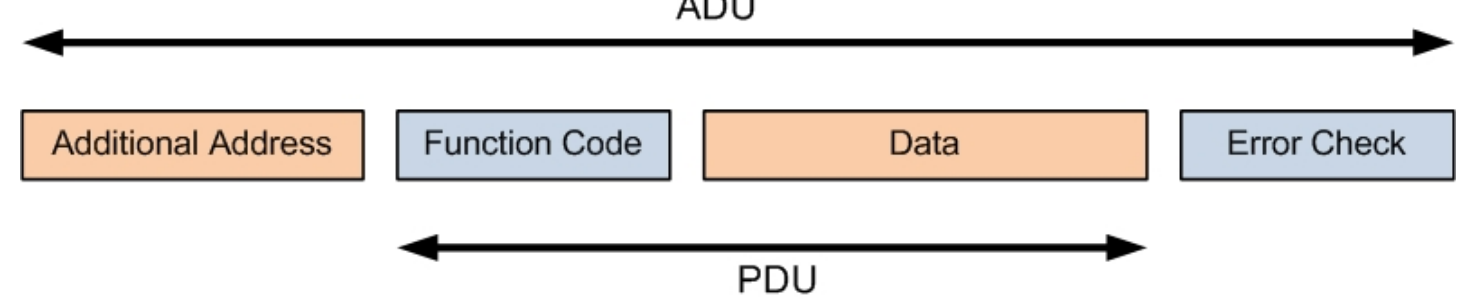

Figure 2.3: MODBUS frame composition

A MODBUS communication session is always started by the master device which sends a message to a specified listening slave device that performs the requested operation and answers to it. Since there can be more devices in the same network, the address in the message header defines the one who should respond to it so that it can be ignored by the others. The MODBUS protocol defines a simple PDU which is kept independent from the underlying communication 
layers. The information, about the specific network on which the MODBUS protocol has to be mapped, can be given by additional fields on the ADU. The master device, initiating a MODBUS communication session, builds an ADU that establishes the format of the request. The function code element tells the slave device what kind of operation has to be performed in order to satisfy the request; sub-function codes can be used for defining multiple actions. The data element contains information to be used to perform the operation specified by the function code. While the function code element must be not empty, the data element can be nonexistent for certain requests. The slave device performs the operation specified by the function code in the request's ADU, and it uses the same value to indicate either an error-free response, by sending back the original function code value, or an erroneous one, by sending back a code equivalent to the original function code but turning its most significant bit to logic 1 .

\subsubsection{Data model}

As every other communication protocol, also the MODBUS has its own data model. It is basically composed by a set of tables with different characteristics. We will consider only the four tables of our interest, which are:

- Discrete input - it is a single bit read-only type that can be provided by an $\mathrm{I} / \mathrm{O}$ system.

- Coil - it is a single bit read-write type that can be alterable by an application program.

- Input register - it is a 16-bit read-only word that can be provided by an $\mathrm{I} / \mathrm{O}$ system.

- Holding register - it is a 16-bit read-write word that can be alterable by an application program.

The four tables can be viewed as one overlaying each other since the difference between inputs and outputs, as well as between bit-addressable and wordaddressable, does not imply any additional application behavior. All the data handled via MODBUS has to be located in the device application memory, but it 
does not mean that the physical address memory has to match the data reference; the only constraint to be satisfied is that data reference has to be linked with the actual physical address. MODBUS uses unsigned integer starting at zero as logical reference numbers.

\subsubsection{A MODBUS library in C: libmodbus}

libmodbus is a shared library written in $\mathrm{C}$ and specifically developed, under LGPL license version 3, for GNU/Linux and MacOSX. It provides functionalities that allow the development of both slave and master applications in respect to the MODBUS protocol established by Modicon. It allows to implement both RTU and TCP communication, and provides all the MODBUS functions. Moreover, it gives structures for the storage and manipulation of data in respect to the MODBUS data model. 


\section{Chapter 3}

\section{Wireless Networks}

The thesis work aims to integrate wireless systems into process industry and business management. This chapter gives an overview of wireless communication and the most common protocols used for wireless communication.

\subsection{Wireless Communication}

Wireless communication [12] is intended as a transfer of information over a variable distance without wires. It can be performed via radio frequency, short-range and long-range microwave, and short-range infrared communications.

The first applications of the wireless communication were implemented by the Croatian scientist Nikola Tesla (1894) who continued the work started by David E. Hughes (1878) and later by Heinrich Rudolf Hertz (1888) regarding the theory of electromagnetic waves.

\subsection{Wireless Common Protocols}

Since the wireless communication is becoming more and more widespread, many protocols have been developed. This section will give a general overview of the most used wireless protocols and focus on the WirelessHART protocol, which constitutes a primary issue for the thesis work. 


\subsubsection{The IEEE 802}

The IEEE 802 is a family of standards related to Local Area Networks (LAN) and Metropolitan Area Networks (MAN). It is maintained by the IEEE 802 LAN/MAN Standards Commitee, which is divided into several teams handling several sub-families of standards. In the following sections a description of the 802.11 [13] and 802.15 sub-families will be given.

\subsubsection{The IEEE 802.11}

The IEEE 802.11 represents a family of standards created by the IEEE LAN/MAN Standards Committee in order to carry out Wireless Local Area Network (WLAN) computer communication using the $2.4,3.6$ and $5 \mathrm{GHz}$ frequency bands. The 802.11 family is composed by the following standards:

- 802.11-1997 - it was the first wireless networking standard which was released in 1997. It introduced network bit rates of 1-2 Mbit/s and three different physical layer technologies: $1 \mathrm{Mbit} / \mathrm{s}$ infrared, 1-2 Mbit/s frequencyhopping spread spectrum and 1-2 Mbit/s direct-sequence spread spectrum (DSSS). A great innovation brought by this standard was the use of 2.4 $\mathrm{GHz}$ in microwave transmissions, while the previous WLAN technologies used much lower ones (e.g. US $900 \mathrm{MHz}$ ).

- 802.11a-1999 - it improves the original standard by the use of a 52-sub carrier Orthogonal Frequency-Division Multiplexing (OFDM) as physical layer, with a maximum raw data rate of $54 \mathrm{Mbit} / \mathrm{s}$. Moreover it uses the 5 $\mathrm{GHz}$ band for microwave transmissions, which gives a significant advantage considering the $2.4 \mathrm{GHz}$ band overloading.

- 802.11b-1999 - it is much similar to the original standard, but much more used since, even though it has same modulation (DSSS) and frequency (2.4 $\mathrm{GHz}$ ), it grants a maximum network bit rate of $11 \mathrm{Mbit} / \mathrm{s}$ instead of 2 Mbit/s. 800.11b devices use to suffer interferences from other products that operate in the $2.4 \mathrm{GHz}$ band like microwave ovens, cordless telephones and Bluetooth devices. 
- 802.11g-2003 - it is an evolution of the 800.11b standard. Using the same $2.4 \mathrm{GHz}$ band, it grants a maximum network bit rate of $54 \mathrm{Mbit} / \mathrm{s}$ thanks to its OFDM modulation scheme (same as 802.11a). Its hardware is yet full compatible with $802.11 \mathrm{~b}$, even though the overall speed of a $802.11 \mathrm{~g}$ network would be reduced in presence of a 802.11 b device.

- 802.11n - it is a proposed evolution of the 802.11 standard not yet delivered, which aims to significantly improve the maximum network bit rate from $54 \mathrm{Mbit} / \mathrm{s}(802.11 \mathrm{a} / \mathrm{g})$ to $600 \mathrm{Mbit} / \mathrm{s}$. The biggest innovation would be the use of a Multiple-Input Multiple-Output (MIMO) physical layer that implies the use of multiple transmitter and receiver antennas in order to improve the network speed. The $802.11 \mathrm{n}$ standard is intended to ensure compatibility with legacy 802.11 devices.

\subsubsection{The IEEE 802.15}

The IEEE 802.15 represents a family of standards created by the IEEE LAN/MAN Standards Committee and specialized in Wireless Personal Area Networks (WPANs). The 802.15 family main standards are the 802.15.1-2002 and 802.15.4-2003 on which underlie respectively the Bluetooth and the ZigBee technologies that will be described in the following sections.

\subsubsection{Bluetooth}

Bluetooth [14] is a short-range wireless networking technology particularly suited for Wireless Personal Area Networks (WPAN) and based on the IEEE 802.15.12002. It is a protocol for exchanging information from both fixed and mobile devices over a short distance. The word Bluetooth comes from the Danish Blåtand, which was the name of the tenth-century king Harald I of Denmark and Norway. As well as Harald I united the Scandinavian tribes into a single kingdom, the Bluetooth technology is intended to unite different communication protocols, making one single standard. All Bluetooth devices operates in the $2.4 \mathrm{GHz}$ band and the protocol provides a maximum network bit rate of $3 \mathrm{Mbit} / \mathrm{s}$, even though Alliance technologies has proposed the WiMedia, which could be capable of providing a maximum bit rate of $480 \mathrm{Mbit} / \mathrm{s}$. 


\subsubsection{ZigBee}

ZigBee [15] is a set of high-level communication protocols based on the IEEE 802.15.4-2003 standard, suited for low data rate WPANs. It aims to provide a simpler and less expensive specification than other existent WPANs (e.g. Bluetooth). ZigBee operates in the industrial, medical and scientific radio bands. Typical application areas include home entertainment and control, mobile services, commercial building and, as in our case, industrial plant management. The standard specifies the physical, MAC and data link protocol layers. Concerning the physical layer, ZigBee uses direct-sequence spread spectrum (DSSS) like some standards of the IEEE 802.11 family. ZigBee has been developed to add mesh networking to the IEEE 802.15.4-2003. It is particularly suited for embedded systems where reliability and versatility are more important than the bandwidth.

\subsubsection{WiMax}

Worlwide interoperability for Microwave Access (WiMAX) [16] represents a telecommunication technology based on the IEEE 802.16 family standards. Its final release operates in the $10-66 \mathrm{GHz}$ band range, but it still supports the 2-11 GHz. The physical layer also differs from the 802.16 family since WiMAX uses a Scalable OFDMA (SOFDMA) instead of the normal OFDMA. WiMAX provides a maximum network rate bit of $3 \mathrm{Mbit} / \mathrm{s}$ and is particularly suited for connectiong $\mathrm{Wi}-\mathrm{Fi}$ hotsposts to Internet and provides a wireless stable alternative to cable broadband access.

\subsection{WirelessHART}

The HART Communication foundation (HCF) released the new HART 7 specification on September 2007. HART is a master/slave protocol which means that a field (slave) device only acts when called by a master. The HART protocol can be used in various modes for communicating information to/from smart field instruments and central control or monitoring systems. The HART 7 specification includes WirelessHART [17], the first open wireless communication standard 
designed specifically for industrial environments in which plant applications need reliable, secure and simple wireless communication. The protocol gets its power from reliability and simplicity of installation and maintainance of its architecture, compared to legacy star-connected systems. WirelessHART supports also legacy HART devices by the use of WirelessHART adapters. We selected WirelessHART as wireless protocol for our purposes since devices at Boliden Plant are HART devices, easily connectible to a WirelessHART network by using the previously mentioned adapters. This is not the only reason. In fact WirelessHART has been designed to meet the unique requirements of wireless networks operating in process plants through the following key capabilities:

- Reliability even in presence of interference, thanks to mesh networking, channel hopping and time-synchronized messaging.

- Security and privacy for network communications through encryption, authentication, verification, key management and other open industry-standard best practices.

- Effective power management through Smart Data Publishing and other techniques that make low-power options practical for wireless devices.

\subsubsection{The WirelessHART Architecture}

A WirelessHART network (fig. 3.1) consists of:

- WirelessHART network manager - it is an application responsible for the management of the network and the connected devices. It creates the network, allows new devices to connect to it, sets the communication schedule for them and monitors every happening in the network. It does not have to reside in the actual plant network, but rather on a gateway device or on other systems device.

- WirelessHART field devices - a field device can be:

- Router device: it is used to improve network coverage and extension by forwarding messages from/to other network devices 
- Process connected instrument: it typically represents a measuring or positioning device used for process monitoring and control. As well as the router device, it is also capable of forwarding messages from other network devices

- WirelessHART Adapter: it allows a HART instrument without wireless capability to be connected to a WirelessHART network.

- WirelessHART gateway - it allows the exchange of data between the mesh network and a plant automation network. It is responsible for providing the access to the WirelessHart devices by a host application or system.

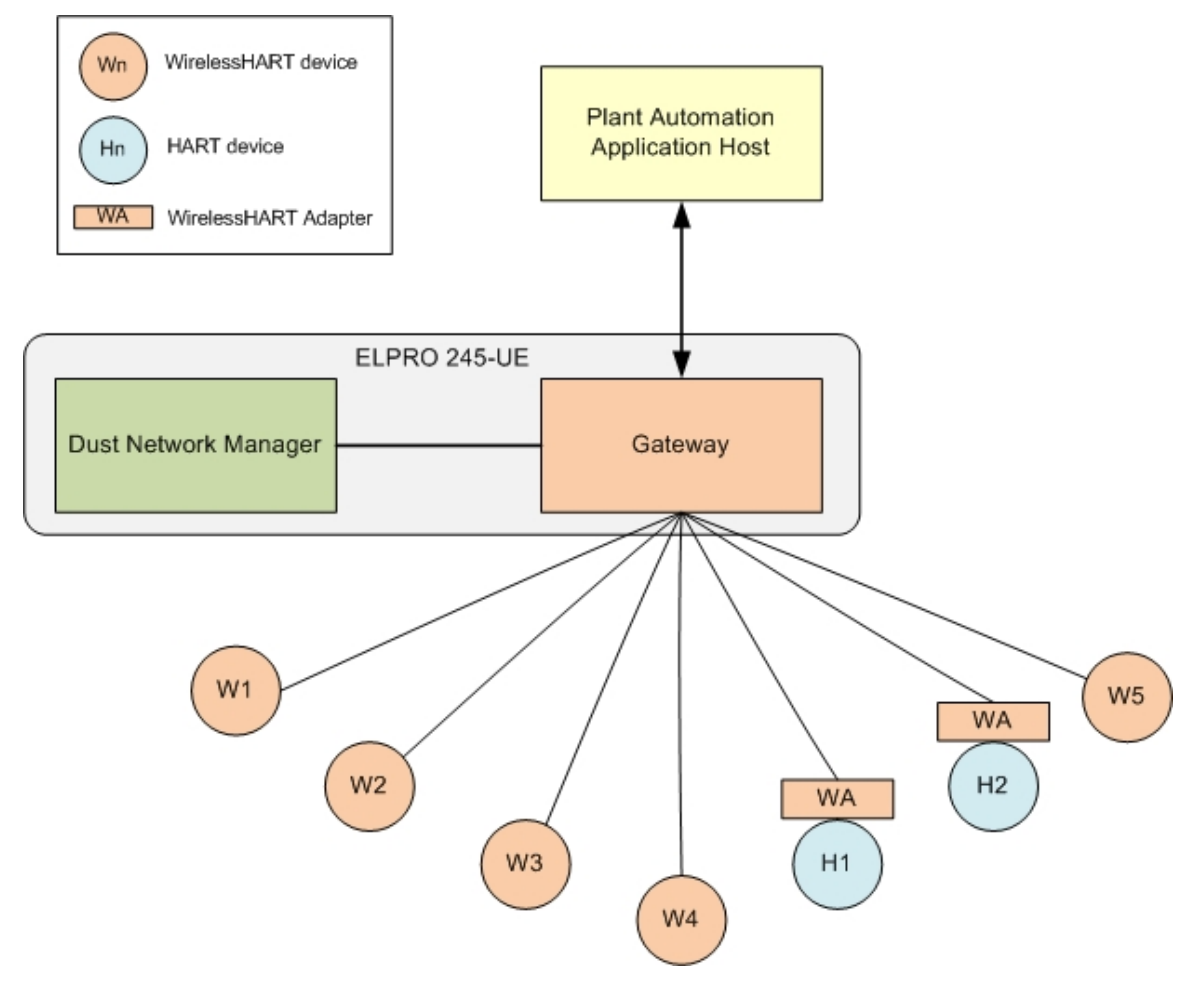

Figure 3.1: A WirelessHART Network

The communications pass always through the gateway that routes the packets to the specified destination using standard HART commands. The plant automation application host might be a TCP network, a remote I/O system or a specific 
bus. The WirelessHART network ensures correct order in transmissions by the MAC protocol, which specifies the communication scheduling for the devices.

\subsubsection{The WirelessHART Network}

The WirelessHART network can be built following several different topologies in order to be able to support a large variety of application requirements. The following three topologies (fig. 3.2) are, however, the most used:

- Star - this type of network is widely used for small applications and it contains only one router device which communicates with the other end devices.

- Mesh - in this type of network, all the field devices are router devices. This provides a robust network with redundant data paths which facilitate fast adaptation of the network to changes of the environment.

- Mixed Star/Mesh - it is a combination of the star and mesh topologies and consequently it will be composed by multiple sets of router devices and end devices.

Star Network

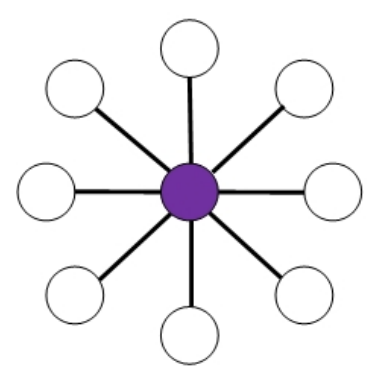

Mesh Network

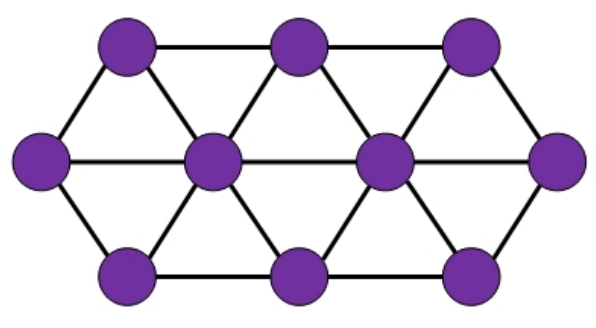

Router Device Node

End Device Node
Mixed Star/Mesh Network

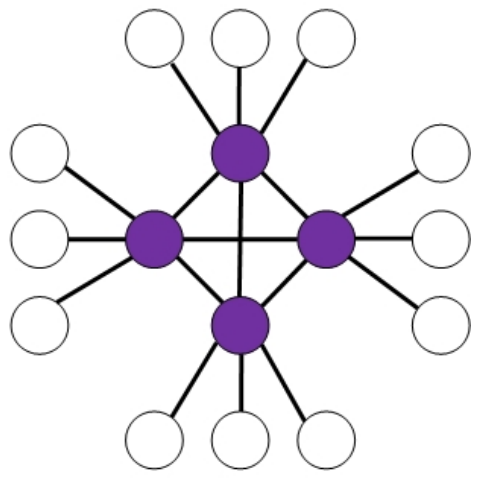

Figure 3.2: WirelessHART Network Topologies 
One of the noteworthiest attributes of a WirelessHART network is its selforganizing ability, which is carried out through three network formation steps: advertising, joining and scheduling. A new network starts with the gateway device sending advertising packets which announce the presence of the network. The packets include time synchrionization information and a network unique ID which has to be matched by the devices trying to join the network. After matching the ID, the new device sends a join request packet to the network manager through the node from which it received the advertising packet. The network manager replies by either accepting the device, through sending back an activation packet to the device node, or refusing it. In case of acceptance, the network manager sets up the links between the new device node and the already existing ones. As last step of the network formation process, the network manager requests information from the new device on how often it will need to send data over the network, and creates a communication schedule for the new device and all the involved intermediate routing devices.

\subsubsection{The Dust Network Manager}

Dust Networks [18] is a company which focuses on design and manufacture of wireless sensor networks for industrial solutions. It was founded in 2002 by Kris Pister, who started the Dust project in 1997 at UC Berkeley, in collaboration with a team of dedicated engineers. Dust Networks' embedded wireless networking products (SmartMesh) provide customers with robust wireless informationgathering systems that give better visibility and control of the connected physical world. These products aim to decrease the customers' time to market and to give end users complex sensors and robust wireless information-gathering systems that decrease the installation costs and increase the efficiency of the operations. SmartMesh products are considered particularly suited for industrial monitoring and control solutions that have specific needs concerning high reliability and flexibility. Within the wide range of SmartMesh products, the IA-500 family of standards-based wireless sensors networking systems has been chosen for our project, due to its compatibility with the WirelessHART standard. These products combine the Dust Networks' robustness together with the industry-leading 
low-power radio technology to provide complete embedded WirelessHART solutions. A SmartMesh-enabled network is composed by a network of motes (remote sensors), a SmartMesh manager that configures and manages the network and client applications that interact with the network through the manager. The communication between the client application and the SmartMesh manager [19] is based on a mixed XML-RPC/XML protocol.

\subsubsection{XML-RPC Communication Protocol}

XML-RPC [20] is a communication protocol based on XML-encoded remote procedure calls (RPC) over HTTP transport mechanism. It was introduced in conjunction by UserLand Software and Microsoft in 1998. XML-RPC is probably the simplest web service approach and lets the developer to make function calls across networks. The power of this protocol is the combination of three standards, the communication architecture (RPC), the vocabulary (XML), and the transport mechanism (HTTP). XML-RPC is a good way for establishing many types of connection between computers as well as between different devices. In our case it will be used for the interaction between the Dust manager and the communication manager application.

\subsubsection{XML-RPC Protocol Description}

In this section we will go through the XML-RPC protocol in terms of the requests and responses required to invoke computations on a remote device using XML through HTTP. More precisely we will give a description of the XML-RPC library we used to create this kind of connection: $\mathrm{xmlrpc-C}$, which is a programming library for writing XML-RPC servers and clients in C. The RPC architecture on which the protocol is based, implies the presence of two parties: a calling process (client) and a called process (server). The client uses procedures that a server provides at a certain HTTP URL. In our case the Dust Manager will act as server while the communication manager application will act as client invoking procedures that operate on the Dust Network, through the Dust Manager. An XML-RPC message is an HTTP-POST request (fig. 3.3) whose body is writ- 
ten in XML. It contains the name of the invoked method within the XML tag $<$ methodName $></$ methodName $>$.

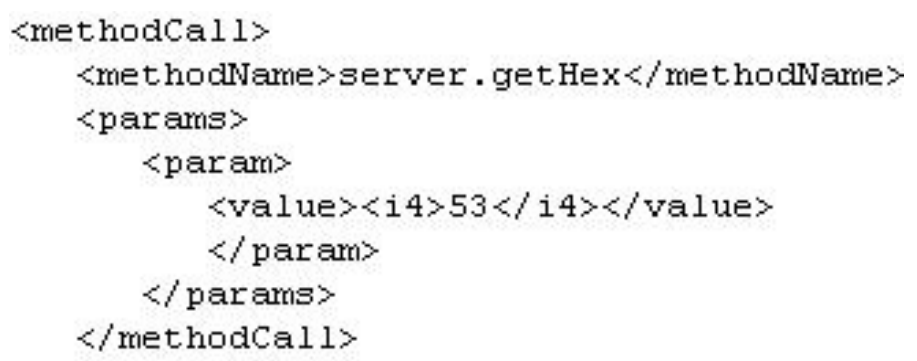

Figure 3.3: XML-RPC Request Example

Procedures usually make use of parameters, specified within the XML tag $<$ params $></$ params $>$ that might in some cases be empty. Parameters can be numbers, scalars, strings, dates as well as complex record and list structures; later in the section we will focus on data-types. In the request presented above the parameter is represented as the data type if (integer). The procedure invoked by the client is executed on the server which replies with another XML formatted message (fig. 3.4).

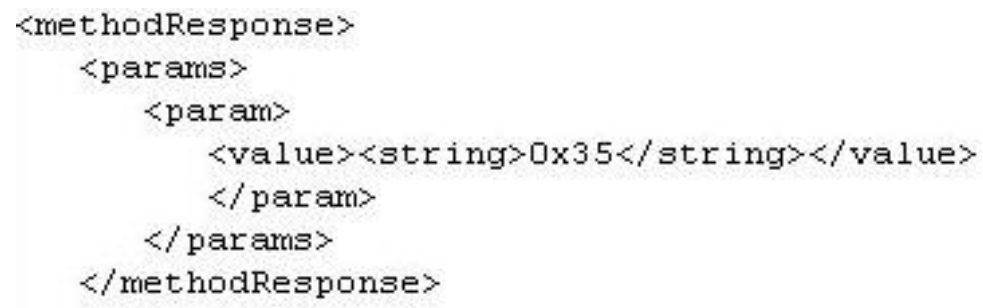

Figure 3.4: XML-RPC Response Example

Requests and responses make use of data types in the form of, respectively, parameters and return values. XML-RPC provides a set of simple data types from which more complex data types can be derived. The simple data types, which are always inserted within the XML tag $\langle$ value $\rangle</$ value $\rangle$, are the followings:

- Integer - it represents the standard integer data type and can represent a 32-bit signed integer. It can be represented both using $<$ int $>n</$ int $>$ or 
$<i_{4}>n</ i_{4}>$ (Note that the number 4 comes from the length of the integer: $4 x 8$ bits). Only numerals from 0 to 9 and the characters + and - , for signed integers, can be used within the tags; no empty spaces are allowed.

- Binary - it is used to represent all those characters prohibited by the XML vocabulary in a base-64 encoding manner. These characters are commonly known as control characters and are the ones with an ordinal value below than the empty space. It is represented by the the binary object, from the encoding defined in RFC 2045 [21], within the tags $<$ base64 $></$ base64 $>$

- String - it represents ASCII string values. There are two different notations to represent strings, either by placing the value within the value tags $(<$ value $>$ sTrInG $</$ value $>$ ) or by explicitly adding a typed representation of strings through $<$ string $>$ s $\operatorname{Tr} \operatorname{In} G</$ string $>$.

- Boolean - it encodes the boolean logic values and it is represented by the tags $<$ boolean $>$ bool</boolean $>$ where bool can have only two possible values, 0 (false) or 1 (true).

- Date-Time - it encodes the date-time type using the ISO 8601 standard. It is represented by an absolute specification of a combined date and time following this pattern:

$<$ dateTime.iso8601>CCYYMMDDTHH:MM:SS</dateTime.iso8601>.

Note that there is no indication about the time zone related to the value.

The basic types are not sufficient for most of the applications. Simple types can be composed into more complex data structures (e.g. arrays, record-like structures):

- Array - it allows synthesis of data items into an untyped list in order to be able to contain elements of different data types. The array type is represented by the tags: $<$ array $><$ data $>\ldots</$ data $></$ array $>$, that will contain the basic data type values (fig. 3.5). 


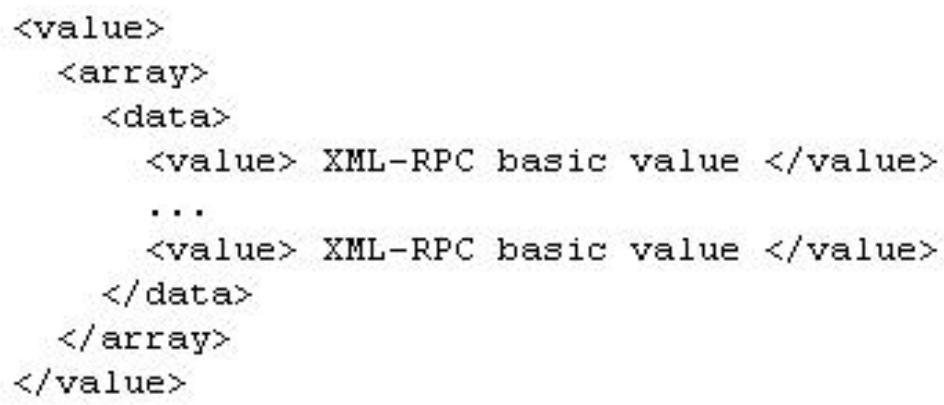

Figure 3.5: XML-RPC Array Type

A data item may be of any XML-RPC type, basic or composed. It is so possible to represent multidimensional arrays by embedding an array within another (fig. 3.6).

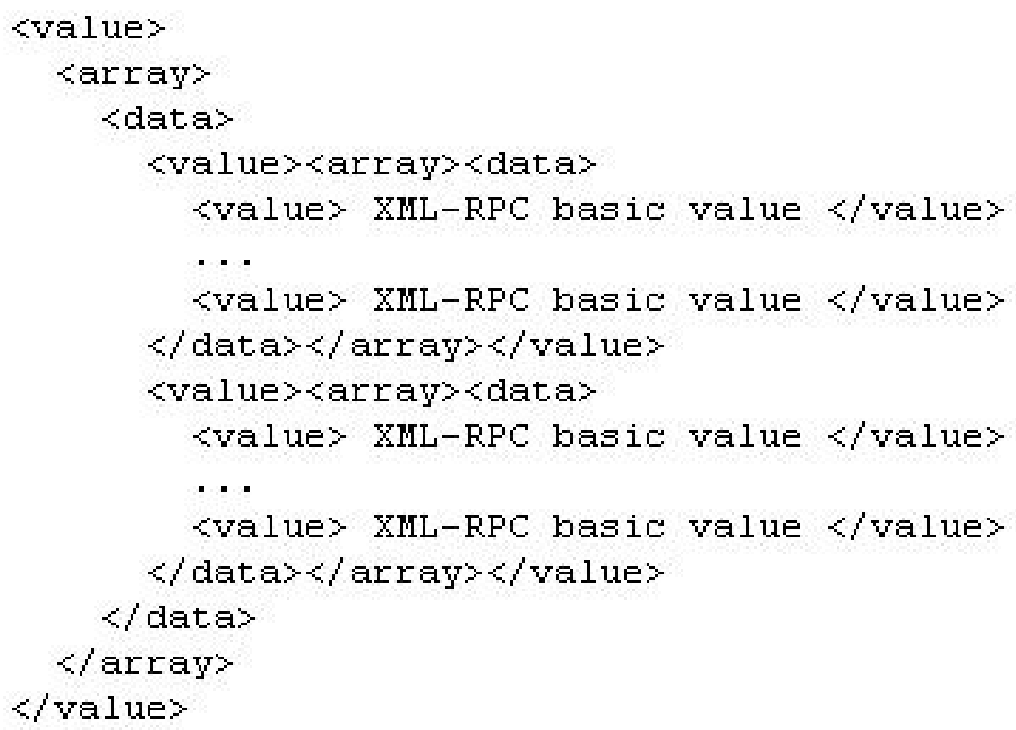

Figure 3.6: XML-RPC Multidimensional Array Type

- Struct - this composed type allows encoding of the associative array, known also as dictionary. It is represented by a set of members, each of which is a pair (name, value). The name is represented by an ASCII string, while the value may be represented by whichever XML-RPC data type, 
both basic and composed. The struct type is represented by the tags: $<$ struct $></$ struct $>$, that will contain the members (fig. 5.3).

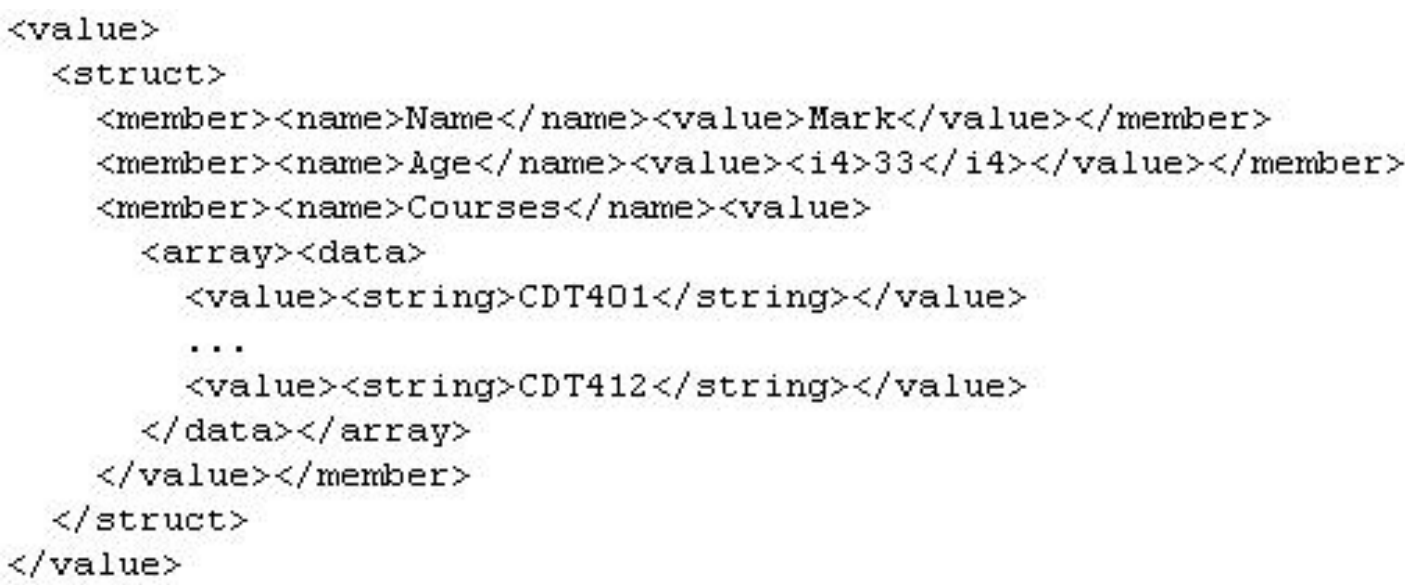

Figure 3.7: XML-RPC Struct Type

\subsubsection{A XML-RPC library in C: xmlrpc-c}

xmlrpc-c [22] is a modular implementation of XML-RPC for C and $\mathrm{C}++$, specifically designed for Unix platforms, which helps programmers to develop XMLRPC both client and server applications. The function libraries that compose xmlrpc-c let developers write applications able to make XML-RPC calls (client) or execute them (server) at any of the levels of understanding of the XML-RPC protocol. There are two sets of libraries composing xmlrpc-c:

\section{- C library}

- libxmlrpc: it provides general facilities

- libxmlrpc_client: it provides facilities for client implementations in $\mathrm{C}$

- libxmlrpc_server: it provides facilities for server implementations in C and that are independent of the transport mechanism

- libxmlrpc_server_abyss: it provides facilities for Abyss HTTP server implementations in $\mathrm{C}$

- libxmlrpc_abyss: it provides the actual Abyss server software 
- libxmlrpc_server_cgi: it provides the facilities for CGI server implementations in $\mathrm{C}$ under an arbitrary HTTP server

\section{- $\mathrm{C}++$ library}

- libxmlrpc ++ : it provides general facilities

- libxmlrpc_client++: it provides facilities for client implementations in $\mathrm{C}++$

- libxmlrpc_server ++ : it provides facilities for server implementations in $\mathrm{C}++$ and that are independent of the transport mechanism

- libxmlrpc_server_abyss++: it provides facilities for Abyss HTTP server implementations in $\mathrm{C}++$

- libxmlrpc_server_cgi ++ : it provides the facilities for CGI server implementations in $\mathrm{C}++$ under an arbitrary HTTP server

- libxmlrpc_server_pstream ++ : it provides facilities for implementing a pseudo-XML-RPC server in $\mathrm{C}++$ that uses a packet stream instead of HTTP and has multi-RPC client/server connections 


\section{Chapter 4}

\section{Involved ABB's Industrial IT Devices}

\subsection{ABB's Industrial IT 800xA System}

The Industrial IT Automation System 800xA (fig. 4.1) provides the technology and solutions needed to achieve a competitive advantage by enabling a plant to perform in a smarter, better and safer manner with cost savings. It improves plant productivity by the following integrated core functions:

- Operations - the Process Portal, an intuitive system interface, provides a consistent method for accessing enterprise data and interacting with multiple applications from several different connected workstations, both locally and remotely.

- Engineering - the integrated engineering environment completely supports the lifecycle of the automation project, from planning to commissioning and operation, passing through configuration and library management. This support minimizes system ownership costs.

- Safety - system functions together with operator and engineering tools reduce plant risks through an accurate management of the human factor. 
- Information Management - information management enhances the usefulness of data by collecting, storing and presenting historical, process and business data from all operations.

- Production Management - it provides speed, agility and control needed to respond to increasing production requests by modelling, executing and tracking information associated with the control flow across the plant.

- Asset Optimization - asset optimization software assesses and reports equipment conditions in real-time in order to decrease costly corrective maintenance and optimize maintenance and calibration work flows.

- Control and I/O - a complete suite of standards-based hardware and software provides total plant control. Each controller is provided with a full line of industrial I/O interfaces to meet all plant environments.

- Device Management - the integration of intelligent field devices via fieldbus standards reduces lifecycle costs through significant cost savings in design, implementation and operation of field equipment.

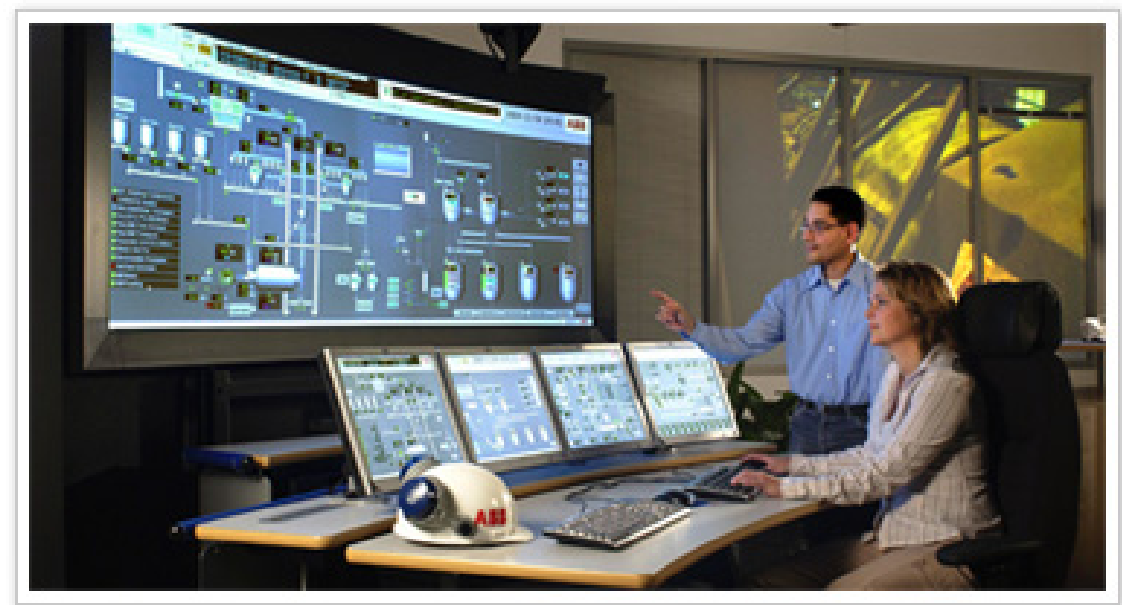

Figure 4.1: A physical view of the 800xA System 


\subsection{ABB's Industrial IT cpmPlus Enterprise Con- nectivity}

The final system will facilitate business management by integrating plant operations handled by the controller device, and a remote enterprise resource planning system (SAP) [23], through the ABB's Industrial IT cpmPlus Enterprise Connectivity (ECS) framework 4.0 [24]. Enterprise Resource Planning (ERP) is a company-wide computer software system used to manage resources, information, and functions of a business from shared data. Integration between plant operations and enterprise business applications can significantly increase productivity, flexibility and quality control. The biggest challenge in this process is dealing with the huge installed base existing in manufacturing today, which has been prohibitive to interface to business management in terms of cost. ECS aims to change this perception by providing a unified framework for managing transactional and event-driven systems in a common environment. The ECS framework provides the basic execution functionality and generic connectivity needed to support configuration and servicing of connectors, by the following set of tools:

- Process Definition Manager - it provides an environment for process definition and development together with support for data mapping and event handling.

- ECS Connectors - it is a collection of ready-to-use enterprise services that provide connectivity to several different types of application:

- Web Services Connectors: it provides integration (fig. 4.2) with enhanced SOA applications.

- Database Connector: it provides connectivity to RDBMS databases.

- OPC Connector: it gives the possibility to connect real-time applications directly to business systems.

- TCP/IP Connector: it provides integration with every TCP/IP based application. 


\subsection{ABB's Industrial IT cpmPlus Enterprise Connectivity}

- XML Connector: it allows processing of an XML structure defined by a given XML schema.

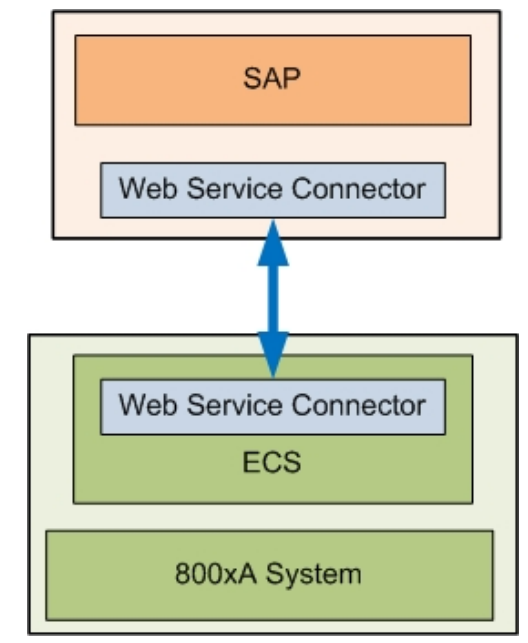

Figure 4.2: ECS Web Service Connectivity

The ECS framework is particularly suited for developing vertical integration solutions between enterprise business management and manifacturing processes. In order to configure the different aspects of a particular integration process, the ECS framework provides a set of tools:

- Process Defition Tool - it allows to model a given process using ISA 95 object models [25]. It uses various connectivity adapters that help to automatically build ISA 95 representation of data structures from several different external systems.

- Report Manager - it generates reports about the process state and history of changes based on the data stored in the ECS database.

- ECS GUI Modeling Tool - it provides functionalities that help in building slim HTML user interfaces, helpful from the final user perspective.

The final product will make use of the ECS framework connectivity in order to share information between the 800xA System and the enterprise business layer 


\subsection{ABB's Industrial IT cpmPlus Enterprise Connectivity}

(SAP). The ECS Process Definition Tool will be used for this purpose. A special Control System plug-in will allow us to import the entire equipment definition from the control layer in the 800xA to the ECS Process Definition Tool. The Process Definition Tool provides also the possibility to check the model correctness by detecting any syntax or inconsistent object naming and definition errors. The created model is saved as a plain XML file so that it can be managed in several ways. After checking the correctness, the tool gives the possibility to generate an 800xA executable code out of the model and to deploy it directly on th 800xA system. After the deployment, it gives to the business management layer the possibility to interact with the executing model through the ECS Web Service Integration. 


\section{Chapter 5}

\section{Integrating the Plant}

In this section we will describe the actual development and setting up of the final product. As mentioned before, the final product is a system which handles the exchange of information between a Wireless HART network and a controller device (ABB's 800xA System) through a communication manager application. In the following sections the development process concerning the communication manager application will be given in details from the early stages of requirements specification to the actual implementation. The final step of the process is a live demonstration of the whole system execution at the Boliden Mining Plant. The SOCRADES project's constraints turned the choice of wireless protocol to Zigbee instead of WirelessHART for the live demonstration. This led to a slightly different scenario from the ideal solution, which uses Zigbee as wireless protocol and a differend communication manager application developed by another member of the project. The future objective is applying the ideal solution to a similar industrial scenario using WirelessHART as wireless protocol and the communication manager application described in this work as wired/wireless interaction manager. 


\subsection{Development Process of the Communication Manager Application}

\subsection{Development Process of the Communication Manager Application}

A development process has to be carried out by following a suited model. In the specific case of the communication manager application, a V-Model (fig. 5.1) has been taken as guide for the development process. The V-Model is an extension of the well-known Waterfall Model. Its main attribute is the strong relationship between development life-cycle phases and the related testing phases. Testing starts from the first phases of the development, helping the developers to take better decisions in order to minimize problems during the final implementation/coding phase, where different design choices could make the difference in terms of time to market and costs. Generally solving problems in the design phases is faster and cheaper than in the implementation phase.

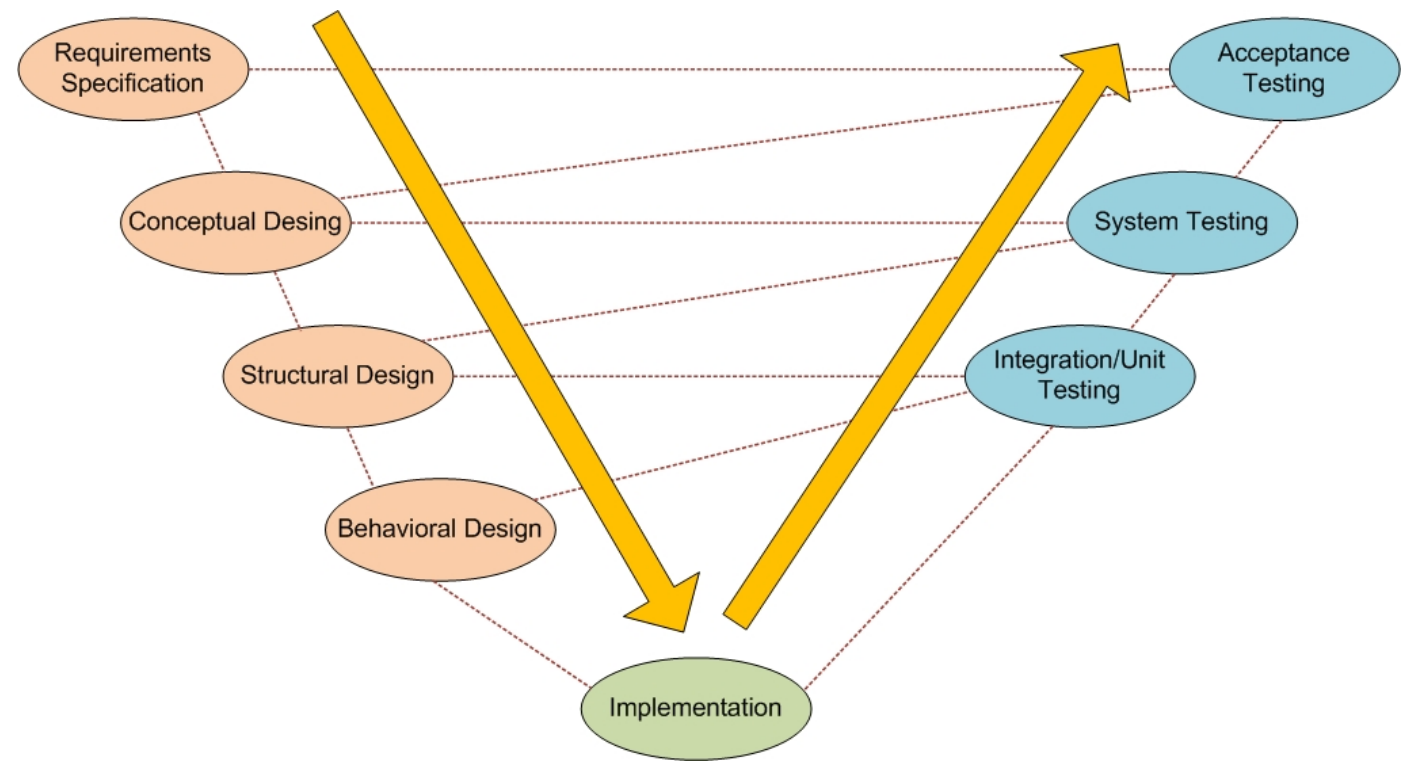

Figure 5.1: Development process V-model 


\subsection{Requirements Specification}

In this section we will go through the requirements specification, which is intended to be a comprehensive description of the aimed purposes for the application under development. It describes in details what the application must be able to do and how it is expected to perform. The requirements specification phase aims to minimize time, effort and cost required by the development process to achieve desired goals. The section is divided into functional requirements specification and extra-functional requirements specification.

\subsubsection{Functional Requirements}

The functional requirements specification is a formal documentation that we will use to describe the application's intended capabilities, from a pure behavioral point of view. It will explain what the application is intended to be able to do and how different components are supposed to interact with each other.

- F1 - Client side in the WirelessHART network: the application will have to be able to act as client in a WirelessHART Network by interacting with the Dust Network Manager.

- F1.1: Connect to the WirelessHART network through the Dust manager. The application has to be able to create, manage and close a connection to the WirelessHART network by interacting with the Dust manager.

- F1.2: Send read requests to the Dust manager. The application must be able to send requests to the Dust manager asking for information about network and connected motes.

- F1.3: Send write requests to the Dust manager. The application must be able to send requests to the Dust manager asking for changings of information regarding network and connected motes.

- F1.4: Receive replies from the Dust manager. The application must be able to receive replies from the Dust manager during both the connection phase and the interaction with the network (read requests). 
- F1.5: Listen for events from the Dust manager. After completing the connection phase, the application must start listening for events coming from the network through the Dust manager. It must be continuously listening until the connection to the network is closed.

- F1.6: Handle events received from the Dust manager. Whenever an event from the network reaches the application side, it must be handled by the application itself in a proper way. This operation might imply operations to be performed by the MODBUS slave side of the application (see F3 - Interaction between the Dust client and the MODBUS slave).

- F1.7: Handle Dust communication errors. The application must be able to handle communication errors since the early connection phase.

- F1.8: Polling requests about the network configuration. The application must be able to keep always updated information about the status of the devices connected to the network by polling requests about the network configuration to the Dust manager.

- F1.9: Keep reliable information about the devices. The application must keep reliable information in the shared memory about the devices connected to the network.

\section{- F2 - MODBUS slave interacting with the 800xA System (MOD-}

BUS master): the application will have to be able to act as slave in a MODBUS connection to the 800xA System, which will act as master.

- F2.1: Listen for incoming connection requests from the MODBUS master device. Once started and connected to the Dust Network, the application must start listening for incoming connection requests from the MODBUS master device.

- F2.2: Handle connection requests from the MODBUS master device. Whenever it receives a connection request from a MODBUS master device, the application must be able to grant the permissions and create the actual connection. 
- F2.3: Listen for requests from the connected master device. Once the connection between the application and the MODBUS master device has been correctly established, the application must start listening for requests from the MODBUS master device.

- F2.4: Handle read requests from the connected master device. The MODBUS master device may send read requests (e.g. polling read requests) to the application, which has to be able to access the MODBUS data memory slot and return the right values.

- F2.5: Handle write requests from the connected master device. The MODBUS master device may send write requests to the application, which has to be able to access the MODBUS data memory slot and perform the requested operation on it.

- F2.6: Handle MODBUS communication errors. The application must be able to handle communication errors since the early connection phase.

- F3 - Interaction between the Dust client and the MODBUS slave: the application will have to be able to perform MODBUS operations while handling Dust events and polling configuration requests.

- F3.1: Listen concurrently for MODBUS requests and Dust events. The application must be able to concurrently listen for and handle MODBUS and Dust requests while polling network configuration requests.

- F3.2: Update MODBUS stored data in response to Dust events. Events received from the Dust manager might imply a modification in the MODBUS data. The application must be able to perform MODBUS data manipulation even during the handling of Dust events.

- F3.3: Ask for updating in the Wireless network in response to MOD$B U S$ write requests. MODBUS write requests from the MODBUS master device might imply a modification in the Wireless network. The application must be able to ask for network, or motes, modification required after MODBUS data changes. 
- F3.4: Initialize the MODBUS data memory slot. Once the application establishes a connection to both Wireless network and MODBUS master, it must be able to initialize the MODBUS data memory slot using the information received from the Dust network configuration.

- F3.5: Handle the MODBUS data memory slot. The application must be able to read/write MODBUS data from a memory slot which will be shared between read and write functions.

- F3.6: Handle access rights to the MODBUS data memory slot. Since the access to the MODBUS data memory slot could be requested by several functions at the same time, the application must be able to manage the access rights.

- F4 - XML handling: the application must be able to handle XML documents.

- F4.1: Parse an XML document. The application must be able to parse and generally handle XML documents since Dust communication is based on a mix XML-RPC/XML protocol.

- F4.2: Recognize Dust notifications. While parsing the XML document, the application must be able to distinguish between event and data notifications from the Wireless network in order to perform the right operations in response to them.

\subsubsection{Extra-Functional Requirements}

The extra-functional requirements specification is a formal documentation that we will use to describe in details the intended quality attributes of the application under development.

- R1: Reliability of data in the shared memory. A reply to every request regarding the network configuration must be received at latest within 1 second, otherwise the data stored in the shared memory cannot be trusted and be available. The system achieves this requirements using an alarming system that is shown in the behavioral design section (fig. 5.11) 
- R2: Shared memory race-conditions-free access. The right to be accessed must be granted at one function at time in order to avoid race-conditions. It is achieved by the system through a mutex lock system on the shared memory that must be used by every function which asks to access the shared memory: if a function is accessing the memory, then it is locked and can not be accessed by another function until the lock is released.

\subsection{Design Specification}

After the completion of the requirements specification phase, the development process continues with the design of the application from both its structural and behavioral perspective. The first step is to create a conceptual design model, which will be the basis for the detailed structural and behavioral models.

\subsubsection{Conceptual Design}

The conceptual design phase aims to build a model (fig. 5.2) of the application under development, from a conceptual, non-detailed point-of-view. This means that it will show which are the application's main components and how they interact both with each other and with the external world. Since the model represents simply an idea about the application structure, no formal languages have been used to build it.

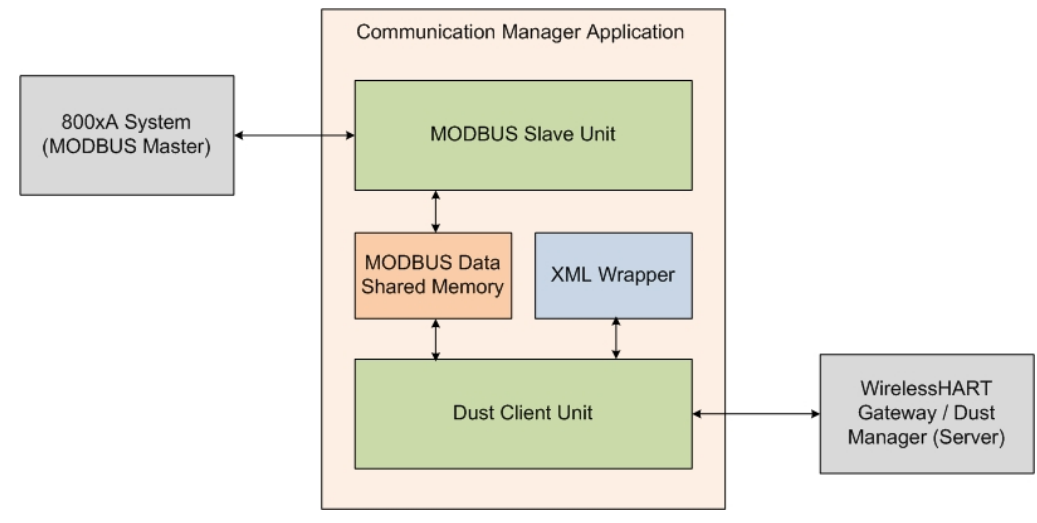

Figure 5.2: Conceptual Model 


\subsection{Design Specification}

Looking at the model, the following elements can be identified:

- Communication Manager Application - this element represents the actual application under development and it can be considered a bridge between the 800xA System and the WirelessHART network. The application is composed by the following components:

- MODBUS Slave Unit: this set of variables and functions will compose the MODBUS slave unit that will act as slave in the MODBUS/TCP master/slave connection to the 800xA System (master device).

- Dust Client Unit: this set of variables and functions will compose the Dust client unit that will act as client in the server/client connection to the WirelessHART network, through the Dust Manager (server).

- MODBUS Data Shared Memory: this set of variables and functions will handle all the issues concerning the shared memory slot for the storage of MODBUS data. It will be used for read/write requests by the MODBUS master (e.g. 800xA System) as well as for the updating operations derived by modifications at the WirelessHART network.

- XML Manager: this set of variables and functions will manage the XML documents used for the communication between the application and the Dust Manager.

- 800xA System - this element represents the MODBUS master device that will be connected to the slave application under development.

- WirelessHART Gateway/Dust Manager - this element represents the Dust Manager that will act as server in the server/client connection between the WirelessHART network and the application under development.

\subsubsection{Structural Design}

Driven by the conceptual model of the application, a more accurate and detailed design phase was carried out: structural and behavioral design using UML as 
modeling language. In this section the structural design will be described in details together with the derived UML design model (fig. 5.3). The communication manager application system is composed by the following components:

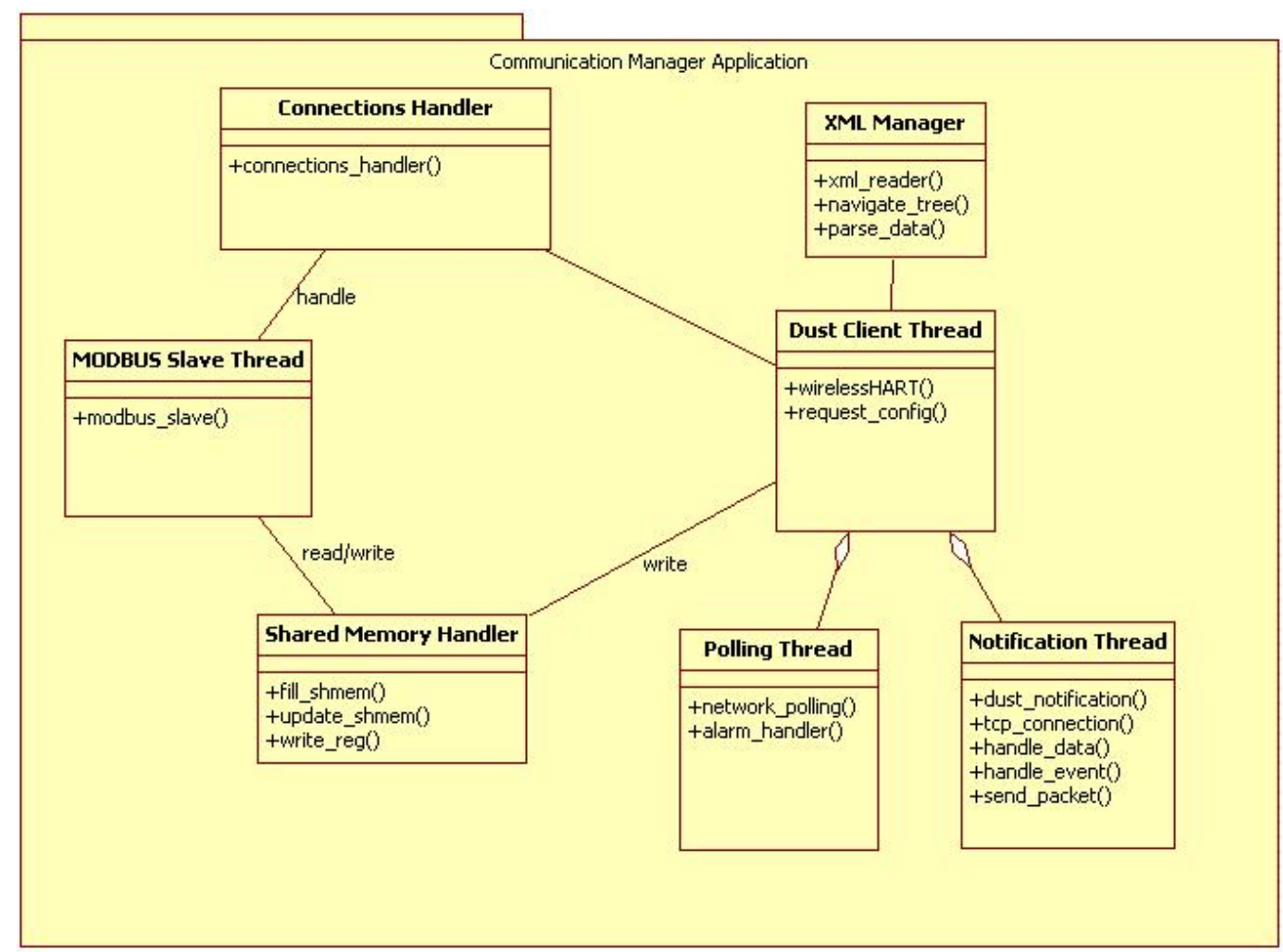

Figure 5.3: Structural model in UML

- MODBUS Slave Thread - The MODBUS slave unit has been thought as a thread in order to be able to perform operations at the same time as the Dust client unit. This design element represents the set of functions and variables that will compose the MODBUS slave thread, responsible for the communication with the MODBUS master devices. It directly interacts with the Connections Handler, which handles the connections, and the Shared Memory Handler, which handles the MODBUS data in the shared memory slot.

- Dust Client Thread - As well as the MODBUS slave unit, also the Dust client unit has been thought as a thread in order to be able to perform its 


\subsection{Design Specification}

operations in parallel with the MODBUS slave unit. The Dust client thread gives birth to two different threads:

- Polling Thread: it is in charge of polling requests to the Dust manager regarding the information about the devices connected to the network in order to have it always updated and reliable in the shared memoery.

- Notification Thread: it is in charge of all the other operations concerning the WirelessHART network management.

- Connections Handler - this element represents the set of functions and variables that handles the two connections: via MODBUS and via Dust.

- Shared Memory Handler - it is composed by a set of variables and functions that are in charge of managing the shared memory slot for MODBUS data about the WirelessHART network.

- XML Manager - it is the set of variables and functions that parses and tokenizes the XML documents in order to extract specific tags and values.

\subsubsection{Behavioral Design}

The natural step after the structural design phase is the behavioral design phase that is driven by the requirements specification previously described. In this phase the expected behavior of the application is formally modelled by using UML sequence diagrams and following what expressed in the requirements specification. Each modelled feature will be mapped on the related functional requirements. The behavioral design phase led to the creation of the following UML sequence diagrams:

- Start Application - this diagram shows the steps performed by the application when it gets started (fig.5.4). The user launches the application which, through the Connections Handler module, connects to the WirelessHART network by the Dust client thread and asks for the configuration of the network in terms of connected motes. The information received by the network will be processed by the XML Manager functions and given back to 
the Connections Handler module which stores it in the shared memory slot properly initialized. After the completion of this operation, the Connection Handler module passes the control to the Dust Client and MODBUS Slave threads which will continue the execution. The functional requirements mapped on this behavioral model are: F3.4, F3.5, F3.6, and F4.1.

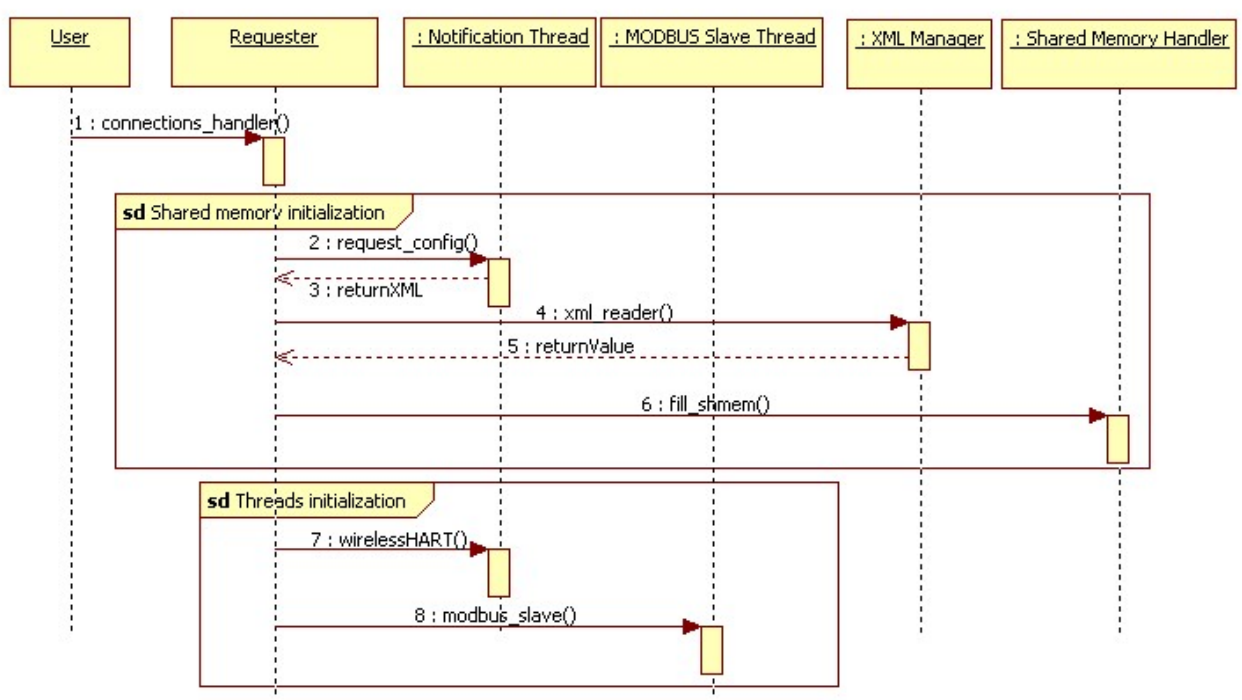

Figure 5.4: Start application sequence diagram

- MODBUS Slave initialization - When the application have been started and the shared memory initialized, the control passes to the MODBUS Slave and the Dust Client threads. This diagram shows how the MODBUS Slave Thread is initialized in order to start its execution (fig. 5.5). The Connections Handler module gives the control to the MODBUS Slave Thread which starts listening for incoming connection requests from MODBUS master devices. When a request arrives, it handles it by either accepting or refusing it. In case of acceptance, it establishes a connection to the MODBUS master device and starts to listen for incoming queries from it. The functional requirements mapped on this behavioral model are: F2.1, F2.2, F2.3, F2.6 and F3.1. 


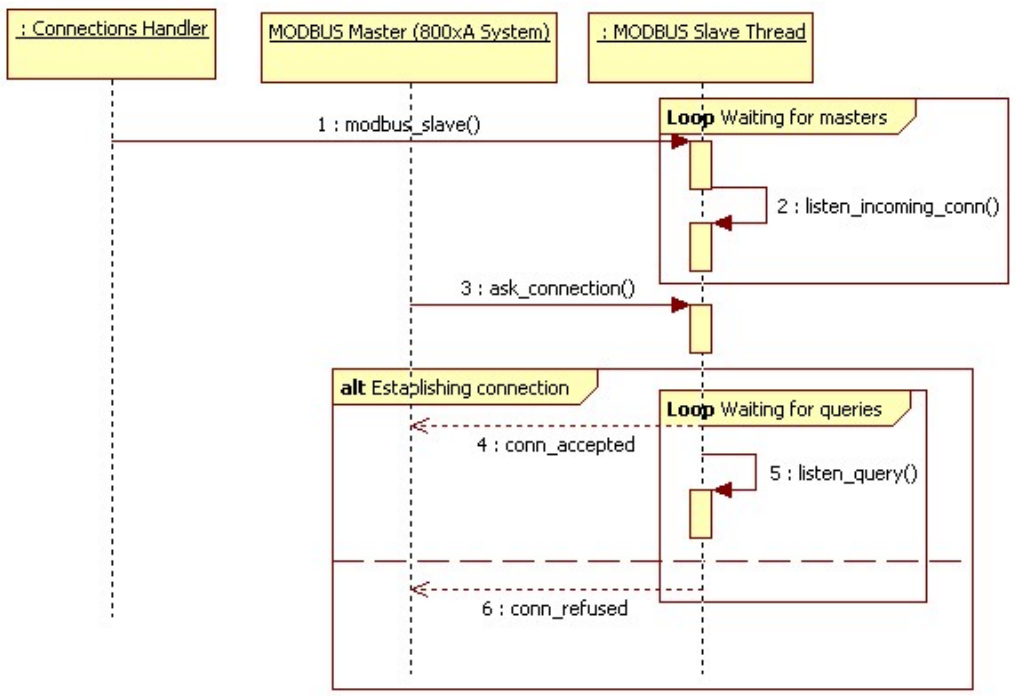

Figure 5.5: MODBUS slave initialization sequence diagram

- MODBUS read request - After established a connection to the MODBUS master device, the MODBUS slave thread module starts listening for queries. This diagram shows how the module handles an incoming read request (fig. 5.6). The MODBUS slave thread module idles by listening for MODBUS queries from the connected MODBUS master device. When a read request arrives, the module asks the permission to read the shared memory slot, and if granted, it reads the required information. The shared memory is not needed anymore, so the module unlocks it and answers the MODBUS master device with either the read values or an error code. The query management has been completed and the MODBUS slave thread module starts again to listen for new incoming requests. The functional requirements mapped on this behavioral model are: F2.4, F2.6, F3.1, F3.5 and F3.6. 


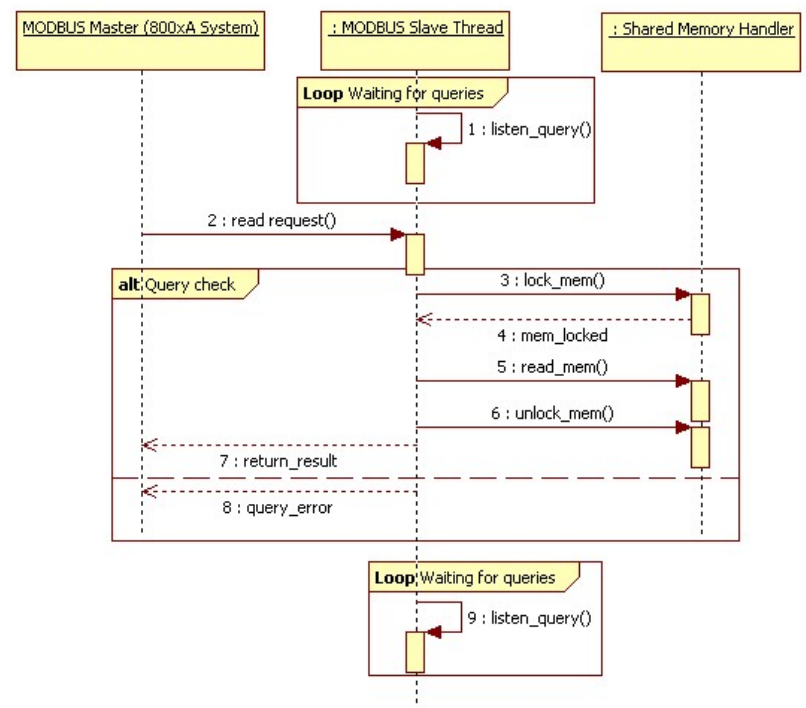

Figure 5.6: MODBUS read request handling sequence diagram

- MODBUS write request - After established a connection to the MODBUS master device, the MODBUS slave thread module starts listening for queries from it. This diagram shows how the module handles an incoming write request (fig. 5.7). The MODBUS slave thread module idles by listening for MODBUS queries from the connected MODBUS master device. When a write request arrives, the module asks the Dust Client Thread module to write the update the network information with the values got from the MODBUS master device. It asks, then, the permission to access the shared memory slot, and if granted, it writes the new values. The shared memory is not needed anymore, so the module unlocks it and in case of errors it sends back an error code to the MODBUS master device. The query management has been completed and the MODBUS slave thread module starts again to listen for new incoming requests. The functional requirements mapped on this behavioral model are: F2.5, F2.6, F3.1, F3.3, F3.5 and F3.6. 


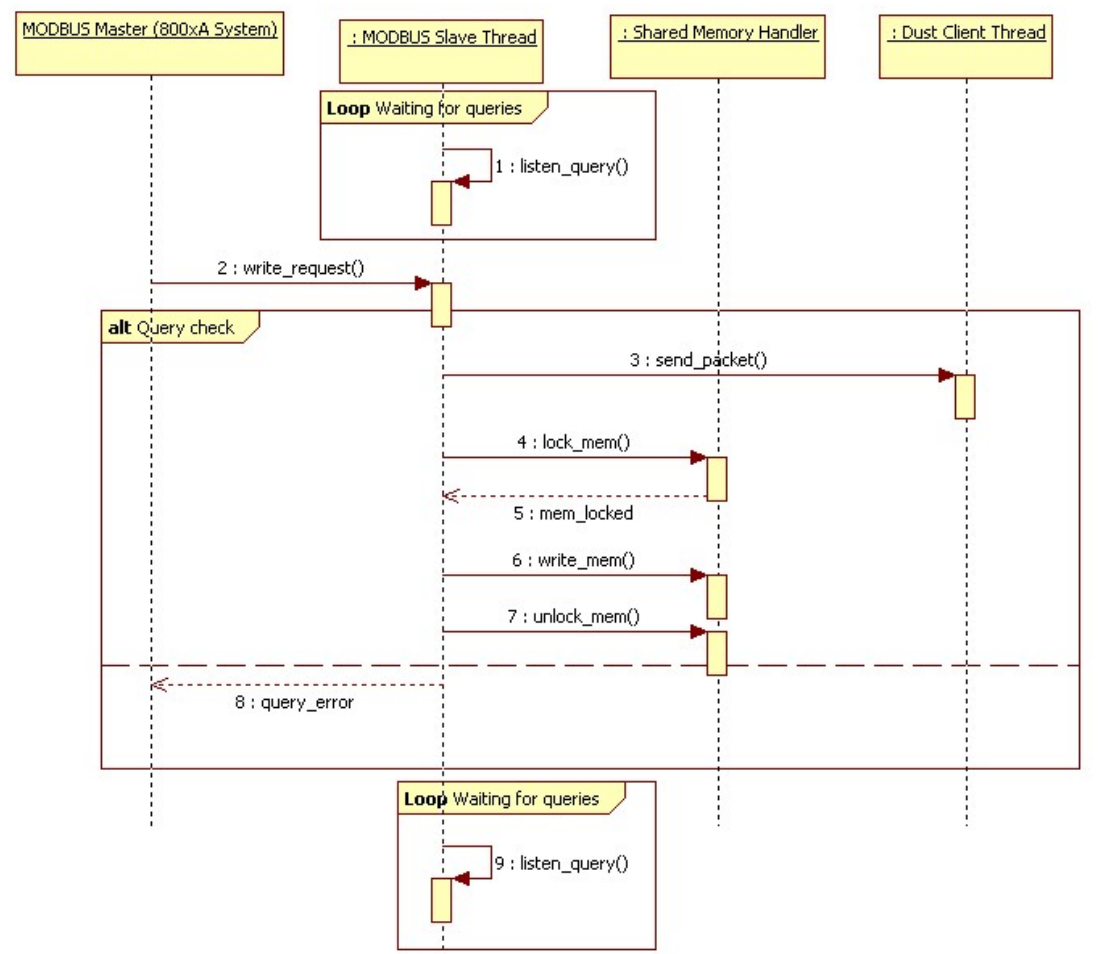

Figure 5.7: MODBUS write request handling sequence diagram 
- Dust Client Thread Initialization - When the application have been started and the shared memory initialized, the control passes to the MODBUS slave and the Dust client threads. This diagram shows how the Dust client thread is initialized in order to start its execution concurrently to the MODBUS slave thread (fig. 5.8).

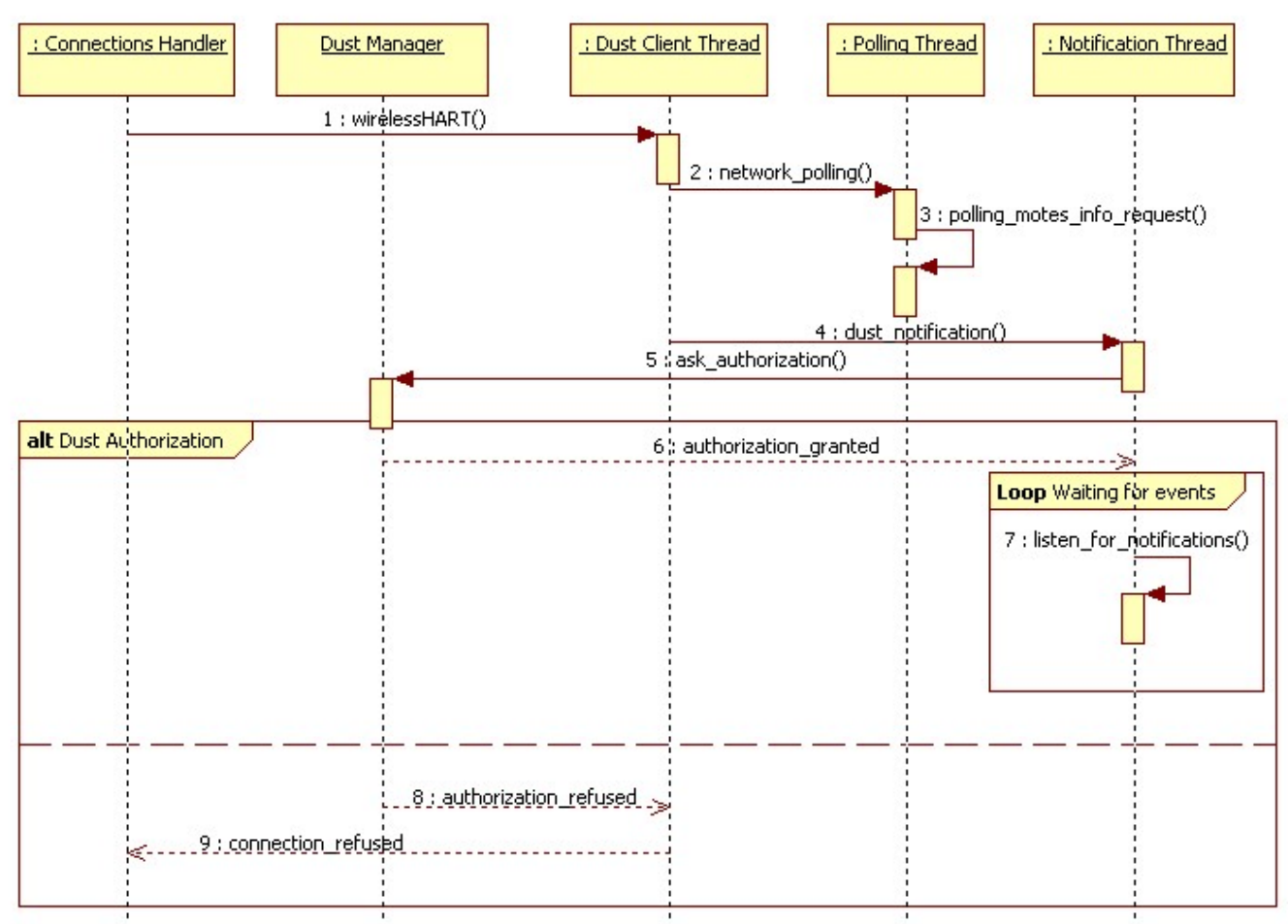

Figure 5.8: Dust client initialization sequence diagram

The Connections Handler module passes the control execution to the Dust Client Thread which, in order to interact with the WirelessHART network, has to connect to the Dust network manager. This connection is performed by two threads: the Polling and the Notification threads. The interaction with the manager starts with an authorization request by the Notification Thread. If the authorization is granted, then the module can start listening for notifications (event/data) from the WirelessHART network, otherwise the Dust client thread can not be started. In the meanwhile, the Polling 


\subsection{Design Specification}

thread starts its job of keeping updated and reliable information in the shared memory regarding the devices connected to the WirelessHART network. The functional requirements mapped on this behavioral model are: F1.1, F1.4 and F1.7.

- Dust Read/Write Requests - The connection between the Dust client module and the WirelessHART network has been established through the Dust network manager. The Dust client can now forward read/write requests to the network. The following diagram shows how the Dust client sends read/write requests to the network (fig. 5.9).

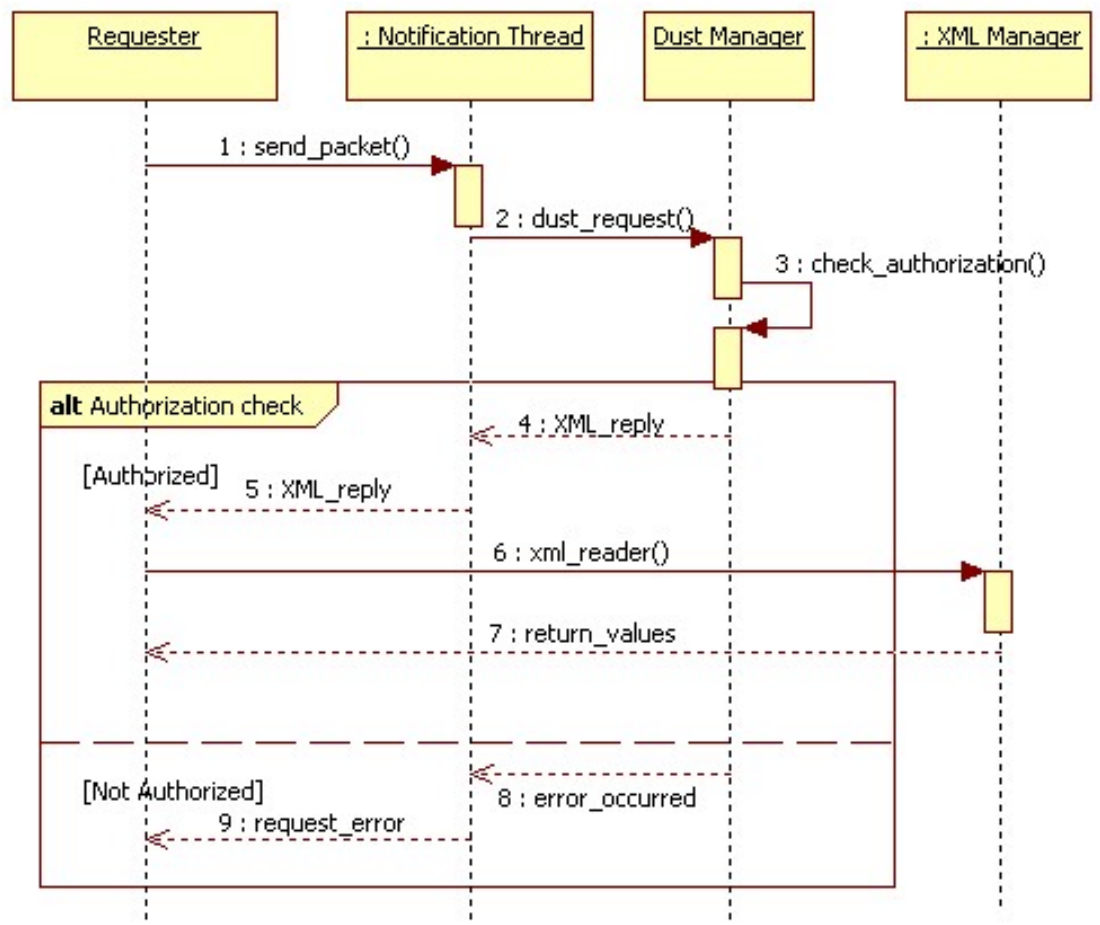

Figure 5.9: Dust read/write request sending sequence diagram

Read requests might be acknowledgments concerning the configuration of the network and the status of the connected devices, while write requests might be modifications at the configuration itself or sendings of packets 


\subsection{Design Specification}

to a specific connected device. The process followed to carry out these operations is exactly the same, except for the contents of the request. In this diagram a requester (e.g. the MODBUS client thread) desires to send a packet to a network's connected device. The Dust client will forward the request to the Dust manager that in order to perform the operation, has first to check the client authorization. If the client is authorized, the manager perform the operation and sends back a reply in XML. The XML document is forwarded, then, to the XML manager module which parses it and returns the actual values to the initial Requester. The functional requirements mapped on this behavioral model are: F1.2, F1.3, F1.4 and F1.7.

- Dust Notifications Handling - The following diagram shows how the Dust client thread module handles incoming notifications coming from the WirelessHART network through the Dust network manager (fig. 5.10). Notifications can have two different shapes: event nofitication, which represents an acknowledgement of some sort of modification occured in the network, and data notification, which notifies a modification of the data related to a connected device. The Dust client module starts listening for incoming notifications right after the connection to the network is correclty established. When a notification arrives, embedded in a XML document, the Dust client module forwards it to the XML manager module that will navigate and analyze the document. Depending on the type of the notification (event or data), it returns the extracted values to the Dust client that might need to request for an operation to be performed on the shared memory slot (note that the operation will imply a shared memory lock/unlock procedure). The functional requirements mapped on this behavioral model are: F1.4, F1.5, F1.6 and F1.7. 


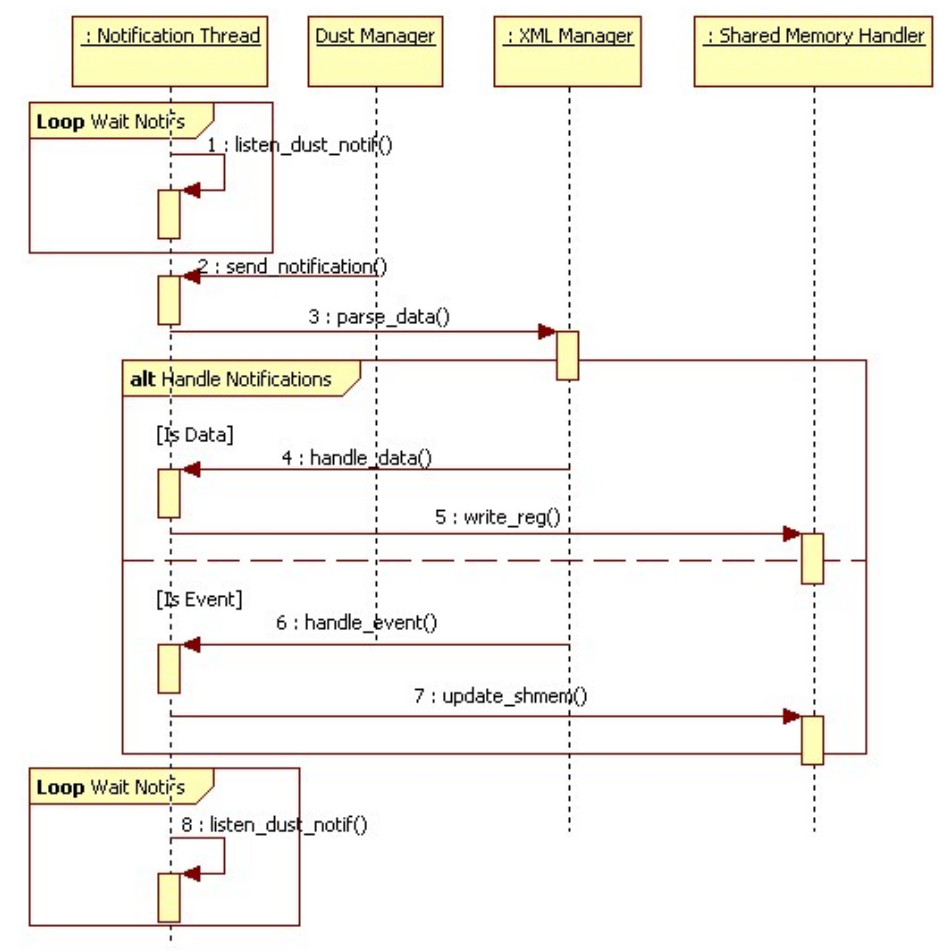

Figure 5.10: Dust notifications handling sequence diagram 
- Polling Devices Information Requests - The following diagram shows how the Dust client thread module keeps reliable and updated information regarding the status of the devices connected to the network. (fig. 5.11).

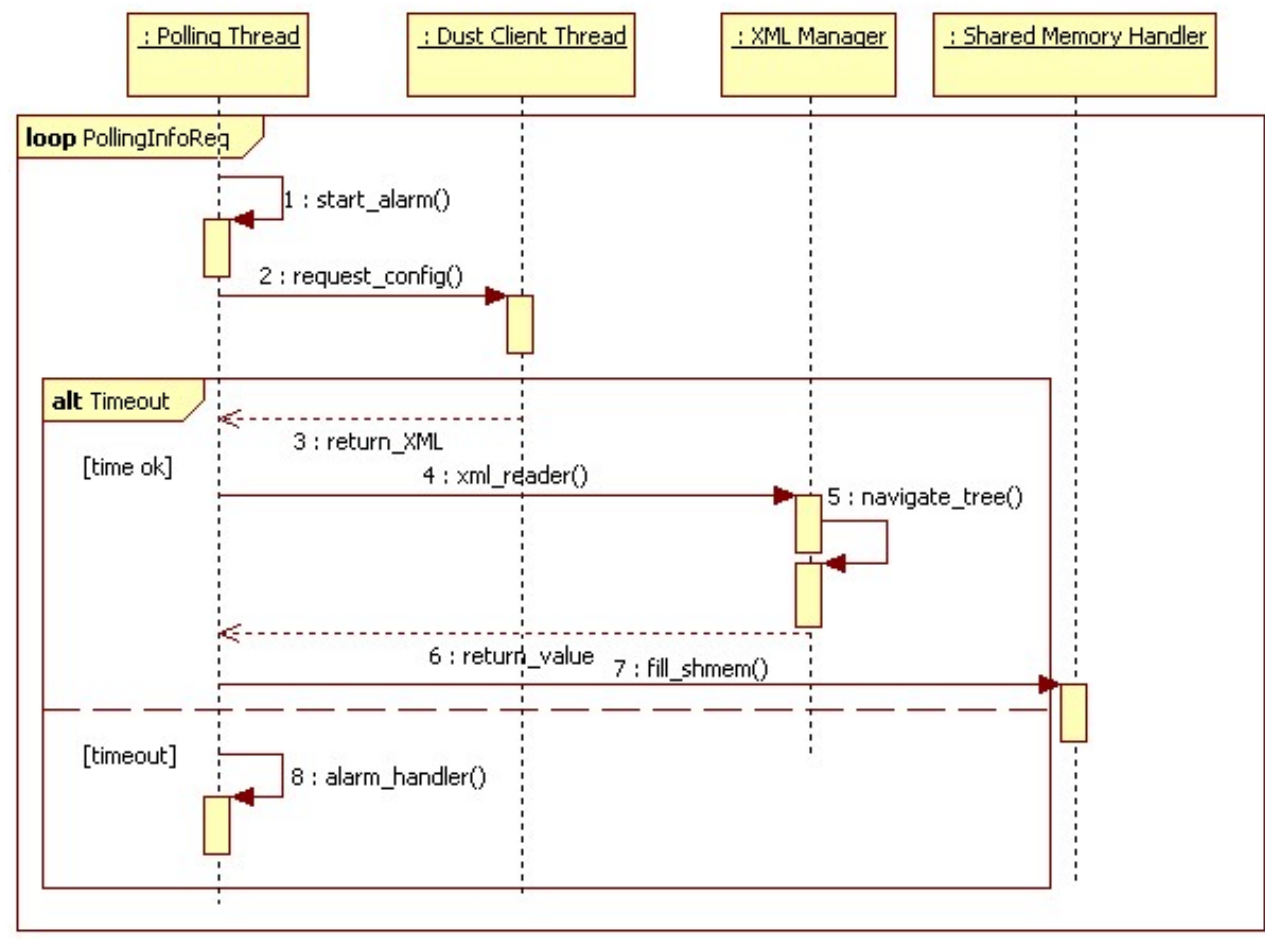

Figure 5.11: Polling Devices Information Requests sequence diagram

The information regarding the devices connected to the network must be always reliable and updated in order to avoid erroneous decisions from the control perspective. The polling thread performs continuosly requests to the network and, when needed, updates the information about the devices. It also ensures reliability of stored information by keeping the replying time for the Dust manager below 1 second. If no reply arrives within that interval, the thread puts the status of every device as 'unvailable' since it cannot ensure correctness of the actual status. The functional requirements mapped on this behavioral model are: F1.7, F1.8 and F1.9. This behavioral model explains how the system implements the extra-functional requirement R1. 


\subsection{Implementation}

The implementation is based on the requirements specification and driven by the design models, both structural and behavioral, described in the previous sections. Due to compatibility and realtimeliness constraints, we decided to develop our application using C, a general purpose programming language. Since its natural environment is represented by UNIX platforms, the development has been carried out on a Linux environment (ver. 7.10 Ubuntu Gutsy). In order to make the application able to interact with the $800 \mathrm{xA}$ Controller system, through the MODBUS master, and the WirelessHART network, through the Dust manager, we needed to include respectively the MODBUS and XML-RPC libraries in the environment, to be used for the code implementation. In the following sections we will focus on the actual implementation of the communication manager application, starting from the development environment setting up and the code implementation.

\subsubsection{Development Environment Preparation}

In this section the procedure to be followed for setting up the development environment will be described in details. In order to be able to develop and execute the communication manager application the following packages have to be installed:

- Linux Ubuntu 7.10 (or newer release) - make sure to have the latest version of the $\mathrm{C}$ compiler/linker gcc and the complete build-essential package.

- zlib - it is a software library written in C used for data compression. In this application the version zlib-1.2.3 has been used. The package can be downloaded from the official website [26] and easily installed by following these steps:

- Unzip the package zlib-xxx

- cd zlib-xxx

- ./configure 
- make

- make install

- libwww - it is a client-side web API written in C and used for both large and small applications as testbed for protocol experiments. The version used for this application was w3c-libwww-5.4.0. The package can be downloaded from the official website [27] and installed by following these steps (note that the zlib library has to be already installed):

- Unzip the package w3c-libwww-xxx

- cd w3c-libwww-xxx

- ./configure -with-zlib

- make

- make install

- xmlrpc-c - it is a modular implementation of the communication protocol XML-RPC for C. The version used for this application was xmlrpc-c1.06.32. The package can be downloaded from the official website [22] and installed by following these steps (note that the libwww library has to be already installed):

- Unzip the package xmlrpc-c-xxx

- cd xmlrpc-c-xxx

.$- /$ configure

- make

- make install

- libmodbus - it is a library for send/receive data between MODBUS devices in respect to the MODBUS protocol established by Modicon. The version used for this application was libmodbus-2.0.0. The package can be downloaded from several sources in the web [28] and installed by following these steps: 
- Unzip the package libmodbus-xxx

- cd libmodbus-xxx

.$- /$ configure

- make

- make install

After completed the installation of the required modules, a last small procedure has to be performed in order to make the system aware of the changes we made on its libraries:

- LD_LIBRARY_PATH=/usr/local/lib/:\$LD_LIBRARY_PATH

- ldconfig

The environment is now ready for the actual code implementation of the communication manager application. The structural design divides the application into five components that can be viewed as collections of variables and functions. In the following sections a description of them will be given from the actual code point of view.

\subsubsection{Connections Handler}

The module described in this section initializes the application and starts the MODBUS and Dust thread modules. The module is composed by the following set of functions:

- void connections_handler() - it initializes the xml-rpc environment variables and creates a connection to the Dust network manager in order to get information about the WirelessHART network configuration. When it receives a reply from the Dust manager, containing the configuration, it uses this information to create a correctly dimensioned shared memory slot and to fill it with the information about the devices which compose the network. The next operation is the initialization of $\mathrm{C}$ mutex that will be used to lock/unlock the access to the shared memory in order to have a race-conditions-free management of it. The function creates, then, two $\mathrm{C}$ 
threads, respectively for the MODBUS and the Dust communications, and passes the control flow to them. If an erroneous state arises during any of the operations, an error message is sent to the console and the application is terminated.

\subsubsection{MODBUS Slave Thread}

The module described in this section operates as MODBUS slave in the connection to the $800 \mathrm{xA}$ system (MODBUS master). The module is composed by the following set of functions:

- void modbus_slave () - it initializes a MODBUS/TCP connection, on the machine IP adress and under the MODBUS/TCP standard port (502), and starts listening for incoming connection requests from MODBUS master devices. When a correct connection request arrives, it is accepted and the function makes the slave thread listen for incoming queries from the connected master, which will be properly handled with by the support of libmodbus library functions. The query might imply a modification to the data stored in the shared memory, and that is the reason for which its handling can be carried out only when the shared memory has been locked through the mutex. The lock will be released at the end of the query execution, and the thread will start again listening for queries. If an erroneous state arises during any of the operations, an error message is sent to the console and the application is terminated.

\subsubsection{Dust Client Thread}

The module described in this section operates as Dust client in the communication between the application and the WirelessHART network, through the Dust Network manager (Dust server). The module is composed by the following set of functions:

- void wirelessHART(const char*) - it creates the two C threads composing the Dust client and passes the control flow to them. 
- void network_polling () - it keeps the information about connected devices updated and reliable by polling a network configuration request to the Dust Manager. If it does not receive a reply from the manager within 1 second, it raises a an alarm signal which is caught and handled by the associated handler function, void ALRMhandler(int).

- void ALRMhandler(int) - it handles unreliable status of the devices by calling the void not_reliable() which sets each device status in the shared memory as unavailable. Then it kills the current erroneous thread and initiates a new one which starts polling again.

- void dust_notification(char*) - it performs all the preliminary operations needed to establish a connection to the Dust manager. When a connection is correctly created, it starts listening for incoming notifications from the network. A notification is embedded in a XML document, so whenever it comes to the Dust client, it is sent to the XML manager module that will take care of its interpretation. If an erroneous state arises during any of the operations, an error message is sent to the console and the application is terminated.

- void handle_data(xmlNode*) - it is invoked by the XML manager module when it interpretes the network notification, forwarded by the Dust client thread module, as a data notification. The function extracts the needed values from the notification XML document and writes them in the shared memory slot if needed. If an erroneous state arises during any of the operations, an error message is sent to the console and the application is terminated.

- void handle_event(xmlNode*) - it is invoked by the XML manager module when it interpretes the network notification, forwarded by the Dust client thread module, as an event. The function extracts needed values from the notification XML document and, if needed, updates the information in the shared memory slot. If an erroneous state arises during any of the operations, an error message is sent to the console and the application is terminated. 
- int tcp_connection(int) - it establishes a TCP connection through C sockets, under to port number specified as parameter by the calling function between the client machine IP address, where the application is running, and the Dust manager IP address. The function returns the socket value that will be used by the caller to perform operations on the TCP connection. It is used by the Dust client thread module since the communication with the Dust manager is done over a TCP/IP connection. If an erroneous state arises during any of the operations, an error message is sent to the console and the application is terminated.

- void die_if_fault_occurred(xmlrpc_env) - it is the function responsible for the error detection and management in the Dust communication. After most of the XML-RPC calls, this function is executed by passing the XML$\mathrm{RPC}$ environment variable as parameter. If it contains an error code, then the function terminates the application and an error message is sent to the console.

- void request_config(char*, const char*) - it performs an XML-RPC call to the Dust manager, asking for the network configuration and more precisely for the element specified as parameter. It receives the answer from the Dust manager in a XML special document, so it extracts the value and then puts it into a global variable visible by the caller function. If an erroneous state arises during any of the operations, an error message is sent to the console and the application is terminated.

- void send_packet(const char*, int) - this function is used when a data packet has to be sent to a specific mote connected to the network. The calling function will invoke it and pass the data packet and the recipient mote ID as parameters. The function asks the Dust manager for the sending packet operation to be performed on the network, and receives a response when the operation has been completed, either successfully or not. If an erroneous state arises during any of the operations, an error message is sent to the console and the application is terminated. Note that functions from the xmlrpc-c library are used for each Dust communication issue. 


\subsubsection{Shared Memory Handler}

The module described in this section is responsible for managing data stored in the shared memory slot. It is composed by the following set of functions:

- void fill_shmem () - it is the function invoked whenever the application is started during the initialization of the shared memory. It asks the Dust manager for the configuration of the network and forwards the XML reply to the void navigate_tree( $\left.x m l N o d e^{*}\right)$ function that extracts needed values from the XML document. If an erroneous state arises during any of the operations, an error message is sent to the console and the application is terminated.

- void navigate_tree(xmlNode*) - it is invoked when a XML document regarding the network configuration has to be parsed in order to extract the values that have to be written on the shared memory. The caller sends the XML document root node as parameter. The function navigates the XML looking for the moteID and state tags whose values will be stored in proper variables and written on the memory by the void update_shmem(int, char*, char*). If an erroneous state arises during any of the operations, an error message is sent to the console and the application is terminated.

- void update_shmem(int, char*, char*) - it prepares data to be written on the shared memory. The caller invokes it by passing the mote ID, its status and the MAC address as parameters. The function calls the void write_reg(int, int) for each value to be written. If an erroneous state arises during any of the operations, an error message is sent to the console and the application is terminated.

- void write_reg(int, int) - it performs the actual writing operations on the shared memory. It is invoked by passing the register index and the value to be written as parameters, so that it is able to correctly write on the shared memory. Note that before to write on the memory, the function locks the mutex, which is released after the completion of the writing operation. 


\subsubsection{XML Manager}

The XML manager module is responsible for handling the XML documents contents. It is composed by the following functions:

- char* $^{*}$ xml_reader(char*, char*) - it tokenizes the XML string passed as parameter and searches for the specified tag. If it finds the searched tag, it returns the value related to it.

- void parse_data(const char*) - it is invoked during the management of Dust notifications. It is responsible for the identification of the notification type (data or event) by navigating the Dust notification XML document. It calls then either the void handle_data $\left(x m l N o d e^{*}\right)$ or the void handle_event(xmlNode*) function that will handle the notification.

\subsubsection{Shared Memory Composition}

In order to be able to correctly read from and write to the shared memory registers, the user needs to know how it is composed. When the application first connects to the WirelessHART network, it asks for the motes configuration and uses that information to initialize the shared memory. In this process a maximum number of motes is retrieved, and 9 consecutive registers for each mote $(9 *$ max Motes in total) are allocated and initialized. The registers will contain the following information:

- 1. Status of the mote - it stores the status of the mote which can be:

- 0: the mote is unavailable for whatever reason explicited in the next register.

- 1: the mote is available.

- 2. State of the mote - it stores the actual state of the mote:

- 0 : the mote is operational. Only in this case the mote is available.

- 1: the mote is lost. The mote is then unavailable.

- 2: the mote is negotiating1. The mote is unavailable. 
- 3: the mote is negotiating2. The mote is unavailable.

- 4: the mote is connected. The mote is unavailable.

- 5: the mote is idle. The mote is unavailable.

- 6: the mote is disconnecting. The mote is unavailable.

- 3. Reason - it stores a reason that explains the state of the mote:

- 0: NONE. The mote is available.

- 1: UNREACH. The mote is unavailable since it is unreacheable.

- 2: NOTCONN. The mote is unavailable since it is not connected.

- 3: CFGERR. The mote is unavailable since there is an error in the configuration of the network.

- 4: MAXMOTES. The mote is unavailable since the network has reached its maximum capacity.

- 4. Payload - it stores the payload assigned to the current mote.

- 5-9. Statistics - they store statistics regarding the mote actions within the wireless network.

Every mote has 9 registers storing the information described above. In order to reach the right register both for reading and writing operations, the number of registers per mote (9) will be used as offset; the actual position $z$ of the register $y$ for the mote with $I D=x$ will then be:

$z=(x * 9)-(9-y)$.

Suppose we have to read the register 2 of the mote with ID $=3$; the actual position will be $(2 * 9)-(9-3)=18-6=12$. 


\section{Chapter 6}

\section{Integrating the Management}

In the Boliden Concentrator Plant the valuable copper $(\mathrm{Cu})$, lead $(\mathrm{Pb})$ and zinc (Zn) minerals are separated from the rock minerals. The final separation phase, called cleaner flotation, generates the final product by varying the different parameters in three discrete recipes: $\mathrm{SA}, \mathrm{SB}, \mathrm{SC}$ which we will call from now on ModeA, ModeB, ModeC. The recipe depends on several factors such as the froth height, the amount of water addition and air flow rate which we want to control. Business elements that may influence the decision on control of the parameters is the preliminary ore quality reported from the different mines supplying it together with the desired quality that gives the best economy in the smelter which represents the customer for the final product. Three points of control have been defined:

1. the ore feed grade of $\mathrm{Cu}$ or added grade of $\mathrm{Cu}+\mathrm{Pb}$ can decide which of the three recipes should be used to maintain the correct final quality

2. the water addition can be increased for a purer quality to the smelter

3. the air flow can be used to control that the final flow is not too high or low, since this would effect the final grade

The enterprise business level application (SAP) can select ModeA, ModeB or ModeC and the control level application interpretes the commands derived from this selection and transfers them into appropriate changes at device level. The devices are connected to the local controller via WirelessHART. In this chapter the 
scenario of the final live demonstration will be given highlighting the plant operator's perspective (ABB's 800xA System) and the actions that can be performed through the business management (ECS) framework.

\subsection{Plant Operator's Perspective}

The Boliden Concentrator Plant is composed by many tanks and sensors of several types. In our scenario we suppose to have two tanks linked in a cascade manner so that whenever the controlled tank's level exceeds its capacity then the adjacent tank starts to be filled by the exceeding flow. The controlled tank is the one placed at the left side in the figure 6.1, which represents the plant operator's workplace developed to ease the execution of local control tasks.

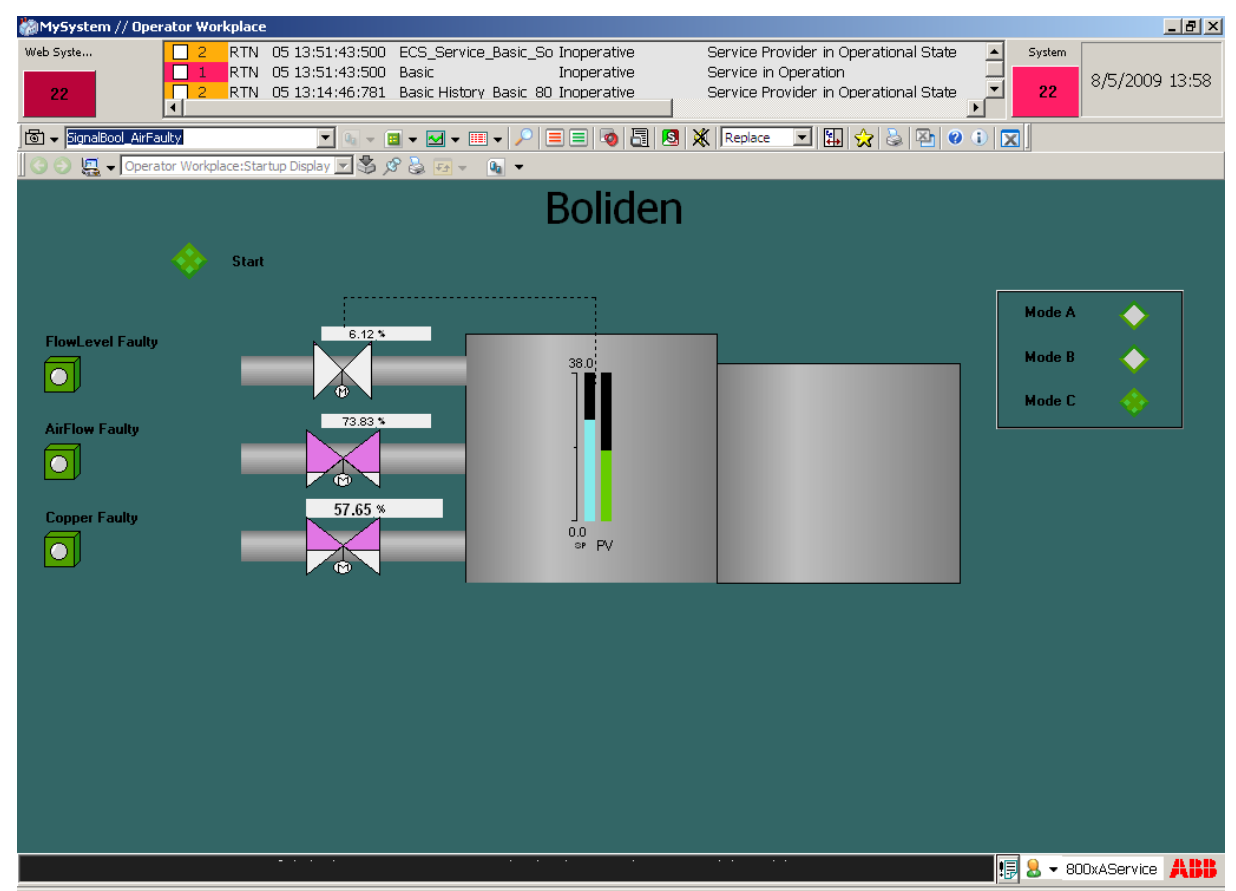

Figure 6.1: Boliden Operator Workplace's view 
Besides the two tanks we can see also three valves, together with their opening level expressed in percentage, that represent, from top to bottom:

- Flow level valve: it represents the control valve which settles the flow level. The operator can check its opening level at any moment through the value in percentage and make adjustments when needed.

- Air flow valve: it represents the control valve which settles the air injection in the tank. The operator can check its opening level at any moment through the value in percentage and make adjustments when needed.

- Copper sulfate valve: it represents the control valve which settles the copper sulfate injection. The operator can check its opening level at any moment through the value in percentage and make adjustments when needed.

In addition to the control valves, there are three control loops, each with a PID controller. The operator will enter a desired level or flow, i.e. set-point, and the PID controllers will manage the valves such that the actual value will correspond to the set-point. Moreover, the flow level actual value can be monitored by the operator through the vertical bars placed on the controlled tank (fig. 6.1). It represents both the flow level value, right bar, and the set point value, left bar. Adjustments at the set point value influence the quality of the product and can be done by switching among the three modes (A-B-C) previously described. The modes status is shown by three visual booleans placed on the right side of the perspective; in the picture we can see that the plant process is running in Mode $\mathrm{C}$ since the related visual boolean is enabled (coloured in green). Another visual boolean placed at the top-left corner, labelled as Start, shows whether the process is running or not. In order to avoid damages at the plant as well as at the product, whenever a sensor (flow, air flow, copper sulfate) detects an error, the operator can point it out by acting on the button placed on the left of the faulty sensor symbol; the process is stopped for further investigation. The value's display of the faulty sensor's value will not visualize a percentage anymore but rather the error symbol $X X X$. This perspective is available as part of the ABB's 800xA System and called Operator Workplace. It has been created using Microsoft Visual Basic that allows to generate a representation of the process, that is the one actually 
deployed on the ABB's 800xA and executed, and which is created and deployed by the ABB's Control Builder M Professional.

\subsection{Enterprise Business Management's Perspec- tive}

The ECS framework will allow business systems to interact with control systems, through the Control System plugin, and make the SAP enterprise business layer able to interact with the process, through the ECS Web Service Integration. The ECS framework deployment in our concrete integration case is made of the following steps:

- Modeling of the integration process - This step takes care of modeling the integration process in a formal representation in terms of ECS ISA 95 objects; this phase is carried out by using the ECS Process Definition Tool. The first operation is the creation of a new integration process model. By the ECS Control System plugin (fig. 6.2) we are able to select the Boliden application running on the $800 \mathrm{xA}$ system and import it in the ECS Process Definition Tool [17]. Moreover, we can select which aspects of the application are supposed to be monitored and visible in the integration model. Methods have been developed in order to allow business management control over the execution process.

- Deployment of the integration process model - this step concerns the deployment of the modelled integration process on the 800xA system. The executable code based on the model is automatically created, and the information about the model are written into the ECS Execution database in order to prepare the persistency layer to store the model state during the execution [29]. The ECS Process Definition Tool automatically checks the correctness of the generated code, but also the correctness of the initial model before the code generation.

- Execution of the integration process model - When the integration model is deployed on the $800 \mathrm{xA}$, we are able to monitor its execution directly 


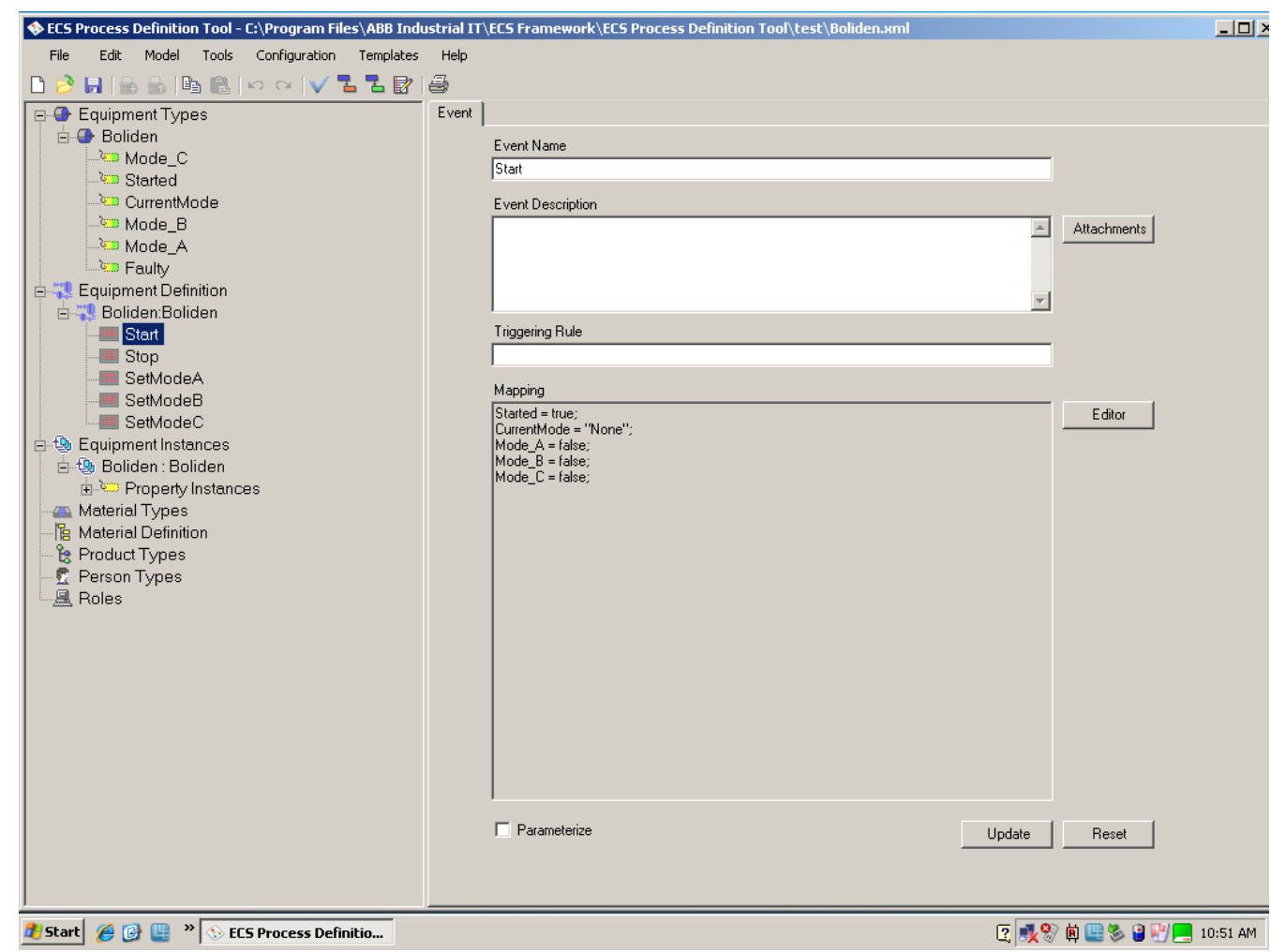

Figure 6.2: ECS Complete Model

through the 800xA Plant Explorer Workplace, as any other 800xA application. In order to make this happen and get the possibility to acquire live data from the execution, the ECS Execution Service connected to the integration model has to be started in the 800xA Plant Explorer.

- ECS Web Service Integration for Business Management - the ECS 4.0 framework provides an integrated web service that allows direct interaction between an enterprise business management layer and the executing model through the Web (fig. 6.3). It gives several possibilities by providing five services:

- ExecuteEvent. It allows to execute an event defined in the executing model by giving the GUID of the instance and the name of the event to be executed as parameters. In our system it will be used for control 


\subsection{Enterprise Business Management's Perspective}

issues by the business layer.

- Ping. It can be used to check the connection to the executing model.

- Read. It is used to read information through parametrized methods defined in the model.

- ReadProperty. It allows to retrieve live data values from the changing properties of the executing model by giving the GUID of the instance and the name of the property to be executed as parameters. It will be used in our system by the business layer to read live values of the executing model properties.

- Write. It is used to write information through parametrized methods defined in the model.

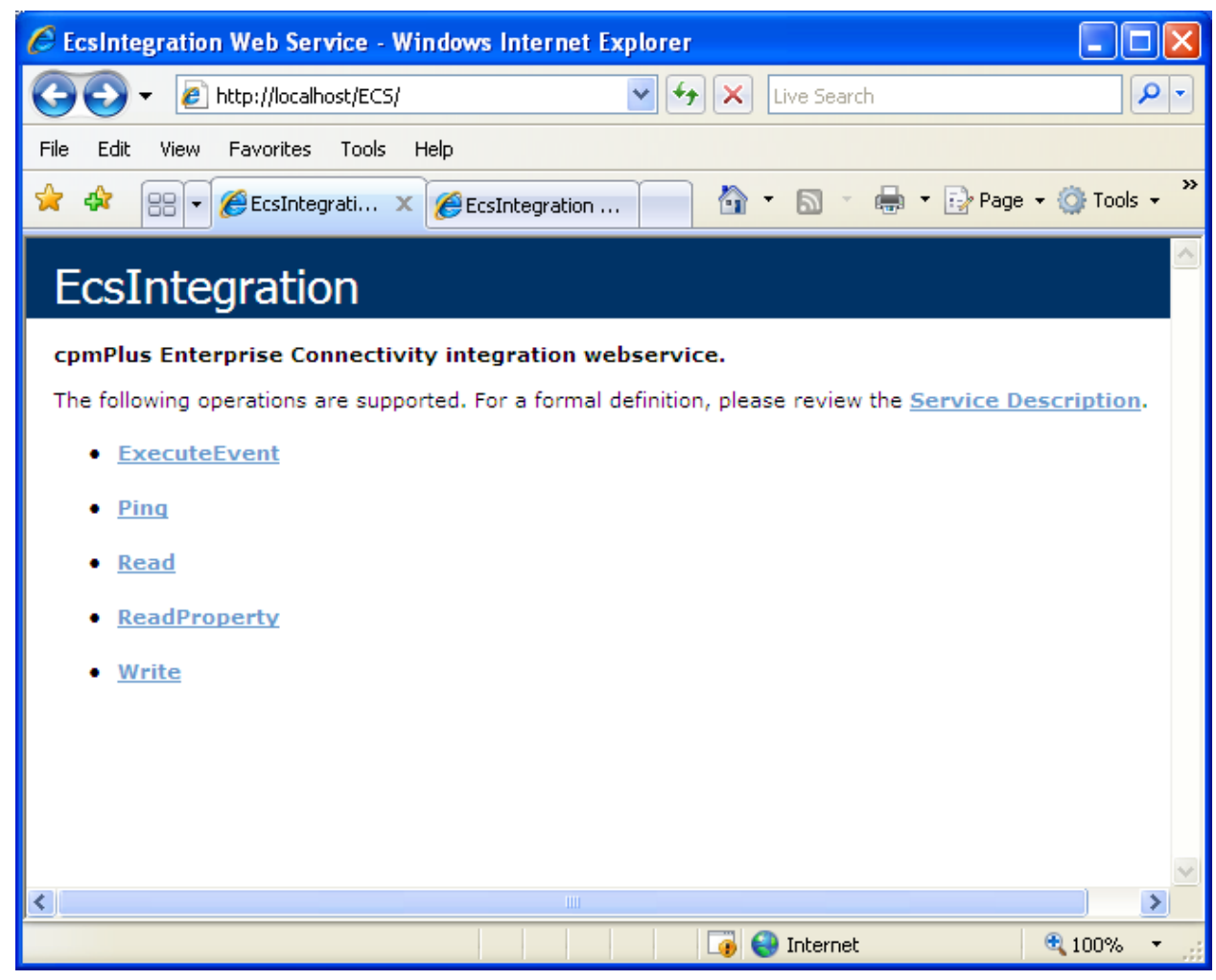

Figure 6.3: ECS Web Service Integration 


\subsection{Enterprise Business Management's Perspective}

We developed the whole ECS model in a way that could allow the business layer, through the ECS Web Service Integration, to remotely get information and manage components in the same fashion as the plant operator can do. Every manipulation performed through the ECS Web Service Integration is immediately reflected on the operator's workplace view and viceversa. The final ECS Boliden Plant model will contain the following components:

- Properties: they are the properties inherited from the control process deployed on the ABB's 800xA System that we want to be able to manage through the ECS Framework and that are available via ECS Web Service Integration through the ReadProperty service by passing as parameters: guid, which represents the process instance in the ECS environment, and propertyName which identifies the property and can be:

- Started: it gives the operational status of the system (on/off)

- Faulty: it gives information about the faulty sensor

- CurrentMode: it gives information about the product quality currently selected for the executing process (Mode A, B or C).

In the table 6.1, a summary of the properties and the meaning of their possible values is presented.

\begin{tabular}{|c|l|}
\hline Property & Value \\
\hline \hline Started & $\begin{array}{l}\text { 0: the system is OFF } \\
-1: \text { the system is ON }\end{array}$ \\
\hline Faulty & $\begin{array}{l}\text { None: system running correclty } \\
\text { FlowLevel: the flow level sensor is faulty } \\
\text { AirFlow: the air flow sensor is faulty } \\
\text { CopperSulfate: the copper sulfate flow sensor is faulty }\end{array}$ \\
\hline CurrentMode & $\begin{array}{l}\text { None: no mode activated } \\
\text { Mode A: system running in Mode A } \\
\text { Mode B: system running in Mode B } \\
\text { Mode C: system running in Mode C }\end{array}$ \\
\hline
\end{tabular}

Table 6.1: Model Properties 


\subsection{Enterprise Business Management's Perspective}

The following pictures show a ReadProperty request (fig. 6.4) and response (fig. 6.5) for the CurrentMode property.

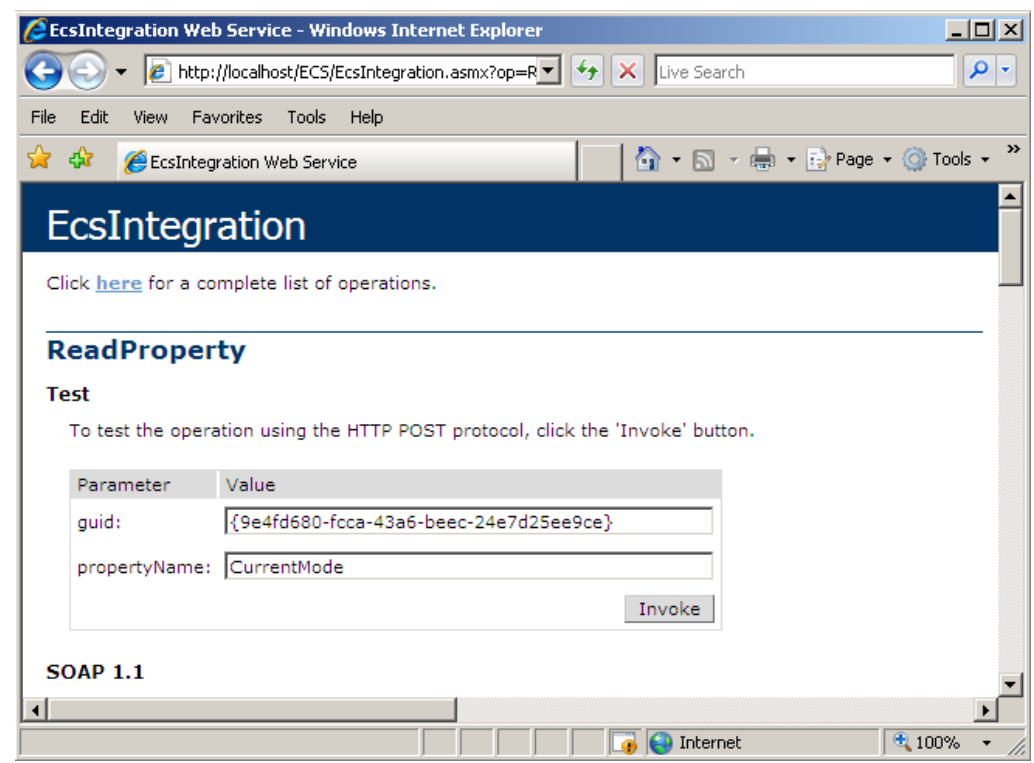

Figure 6.4: ReadProperty Request

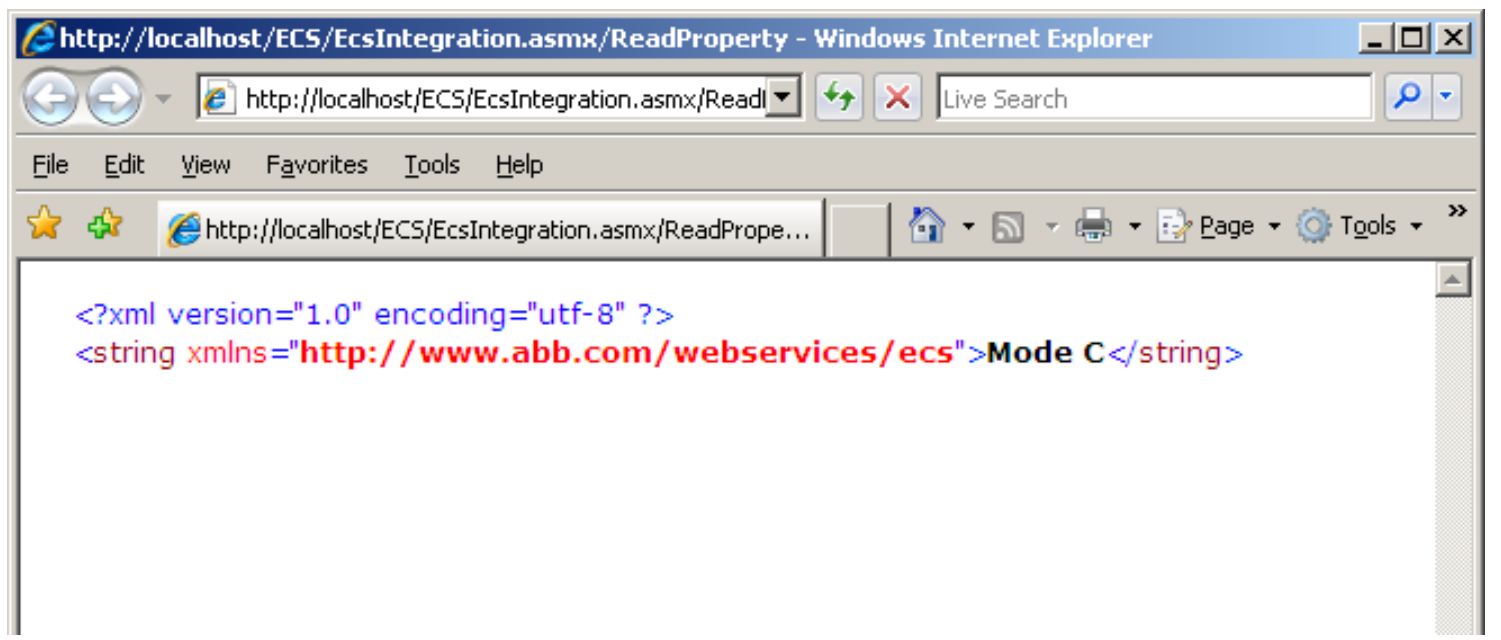

Figure 6.5: ReadProperty Response

- Methods: they allow the actual management of the defined properties. They can be executed remotely via ECS Web Service Integration through 


\subsection{Enterprise Business Management's Perspective}

the ExecuteEvent service and passing as parameters: guid, which represents the process instance in the ECS environment, and eventName which identifies the method to beexecuted and can be:

- Start: it starts the process execution

- Stop: it stops the process execution

- SetModeA: it set Mode A as product quality

- SetModeB: it set Mode B as product quality

- SetModeC: it set Mode C as product quality

In the table 6.2 , a summary of the methods and the meaning of their execution responses is presented.

\begin{tabular}{|c|l|l|}
\hline Method & True & False \\
\hline \hline Start & System has been started & Method execution error \\
\hline Stop & System has been stopped & Method execution error \\
\hline SetModeA & $\begin{array}{l}\text { Mode A has been enabled } \\
\text { and B-C disabled }\end{array}$ & Method execution error \\
\hline SetModeB & $\begin{array}{l}\text { Mode B has been enabled } \\
\text { and A-C disabled }\end{array}$ & Method execution error \\
\hline SetModeC & $\begin{array}{l}\text { Mode C has been enabled } \\
\text { and A-B disabled }\end{array}$ & Method execution error \\
\hline
\end{tabular}

Table 6.2: Model Methods 


\subsection{Enterprise Business Management's Perspective}

The method execution gives a boolean as response: true if the method has been correctly executed, else false. Figure 6.6 shows a ExecuteEvent request for the SetModeC method.

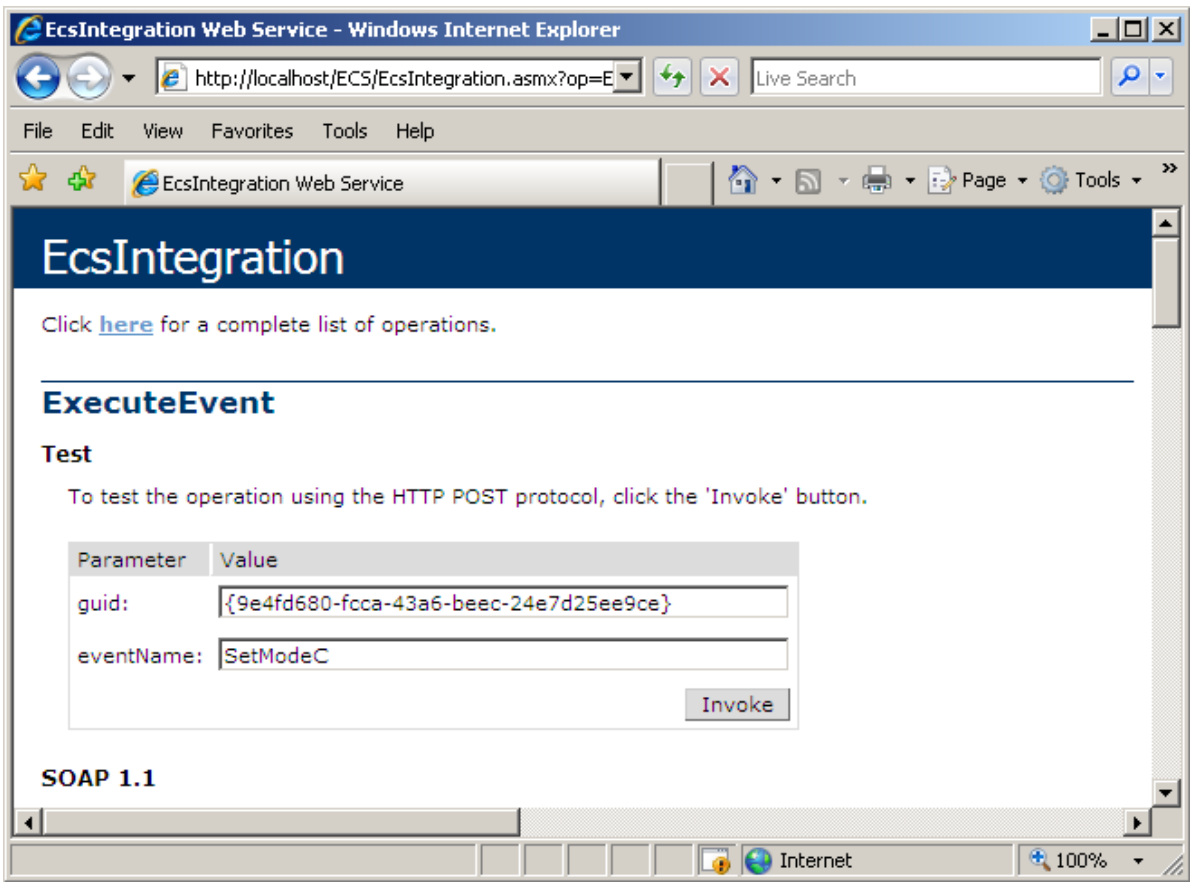

Figure 6.6: ExecutionEvent Request 


\section{Chapter 7}

\section{Conclusions}

The purpose of this thesis work was to create a system that integrates wireless systems into an industrial process and that allows both local control and enterprise business management in a remote fashion. The work gives an ideal solution which comprises the WirelessHART as communication protocol between the sensors' network and the system, but due to the SOCRADES project's constraints, it could not be used in the real-life final demonstration, for which a slightly different system has been developed and also presented. Nevertheless, the ideal solution could be used whenever the WirelessHART protocol results available.

The communication manager application, that is the core part of this work, has been developed in order to handle the communication between a WirelessHART network and wired MODBUS/TCP devices (e.g. ABB'S 800xA System) in an industrial environment. The importance of such application is the capability of making communicate wireless devices that use an industrial de-facto standard wireless protocol, WirelessHART, and wired devices that use one of the most common protocols for industrial wired communication, MODBUS. The power of the product is its usability in any project which needs a communication between a WirelessHART network, which uses a DustNetworks SmartMesh Manager as network manager, and a set of devices communicating via MODBUS/TCP. The future goal will be to use this application in a real-life industrial process such the one presented in this thesis work. The SOCRADES project's constraints turned the choice of wireless protocol to Zigbee instead of WirelessHART. This decision led to a slightly different scenario from the ideal solution, which does not 
comprise the developed communication manager application but rather a similar application, developed by another member of the project, that uses Zigbee as wireless protocol.

In any case, the sub-system regarding enterprise business management and operator workplace, which has been described in the last chapter, would fit also in the case of using the WirelessHART as wireless protocol. The communication application manager will be, then, used as bridge between the wireless sensor's network and the wired ABB's 800xA Controller. This is possible thanks to the high flexibility and adaptability of the developed system's design, that already allowed us to turn to a different wireless protocol, Zigbee, in the very last phases of the SOCRADES project just by making minor modifications. The developed ECS model allows enterprise business systems to interact with the control process in a remote fashion by using the ECS Web Service Integration. The business layer only needs an internet connection so that it can connect to the IP address of the ABB's 800xA System and interact with the control system by following the specification of the ECS model's properties and methods. During the system development several challenges have been faced; the followings can be considered the most noteworthy:

- Wired/Wireless protocols interaction - the communication manager application operates as 'bridge' between the wireless network sensors system and the wired controller. It has been developed in order to respond to both wireless and wired requests and at the same time when needed.

- Reliabilty and security of shared common data - The application provides a secure exchange of information between wireless and wired devices thanks to a race-conditions-free shared memory implemented with a threads mutex lock system, which handles access rights in order to allow only one function at time to access the memory. In order to avoid wrong behaviors of the process which could lead to damages at both product and resources, the application provides a strict timing constraint on the availability of the shared data; whenever a response from the network regarding its configuration does not come within 1 second, the application marks the data as 
unreliable until it gets back a response from the network and reliable data is restored.

- Actual plant loops modeling - in order to be able to execute the live demonstration at the Boliden Mining Plant, the actual process loops have been modeled in the 800xA System and executed to replace the plant's loops.

Further developments for this work would most probably be extensions to other important wired fieldbuses such as PROFINET, PROFIBUS, Fieldbus Foundation, which will make the system usable in most of the industrial processes that need wireless-wired direct interaction. An additional useful feature would be the development of a tool for user-mapping of data, that we provided as a fixed shared memory, between wireless and wired. User will be, then, able to specify which data have to be shared and where to store, in order to have a customized fashion of sharing data. 


\section{Bibliography}

[1] SOCRADES Group. Service-oriented cross-layer infrastructure for distributed smart embedded devices. http://www. socrades.eu.

[2] ABB Automation Technology Products. IndustrialIT 800xA - System (version 5.0): System Guide: Technical data and configuration information. ABB, 2006.

[3] ELPRO Tech. Homepage. http://www.elprotech.com/.

[4] A. S. Tanenbaum. Computer Networks, Fourth Edition. Prentice Hall, 2003.

[5] IEEE 802.3 Working Group. Official website. http://www . ieee802 .org/3/.

[6] Fieldbus Foundation. Official website. http://www.fieldbus.org/index. php?option $\backslash=$ com_content\&task=view\&id=45\&Itemid=195.

[7] A. Pigan and M. Metter. Automating with PROFINET, Second Edition. Siemens, 2008.

[8] MODBUS-IDA Group. Official website. http://www.modbus.org/.

[9] ODVA. EtherNet/IP - CIP on Ethernet Technology: Technology Overview. ODVA, 2006.

[10] ODVA. Devicenet technical overview. http://www.odva.org/Portals/0/ Library/Publications_Numbered/PUB00026R1.pdf.

[11] CANopen Solutions. Official website. http://www.canopen-solutions . com/canopen_fundamentals_en.html. 
[12] D. Tse and P. Viswanath. Fundamentals of Wireless Communication. Cambridge University Press, 2005.

[13] IEEE 802.11 Working Group. 802.11-1999 Information technology Telecommunications and information exchange between systems - Local and metropolitan area networks - Specific requirements - Part 11: Wireless LAN Medium Access Control (MAC) and Physical Layer (PHY) Specifications. ANSI/IEEE Std 802.11, 2003.

[14] Davies A. C. An overview of bluetooth wireless technology and some competing lan standards. ICCSC '02, pages 206-211, 2002.

[15] Zigbee Alliance. Official website. http://www.ZigBee.org.

[16] WiMAX Project. Official website. http://www.wimax.com/education.

[17] J. Song, S. Han, A. K. Mok, D. Chen, M. Lucas, M. Nixon, and W. Pratt. Wirelesshart: Applying wireless technology in real-time industrial process control. In 14th IEEE Real-Time and Embedded Technology and Applications Symposium (RTAS), 2007.

[18] Xmlrpc-C Project. Official website. http://xmlrpc-c.sourceforge.net/.

[19] Dust Networks. SmartMesh IA-510 Manager: XML API Guide. Dust Networks.

[20] S. St. Laurent, J. Johnston, and E. Dumbill. Programming Web Services with XML-RPC, First Edition. O'Reilly, 2001.

[21] RFC2045 Standard. Official website. http://www . ietf .org/rfc/rfc2045. txt.

[22] Libwww Project. Official website. http://www.w3.org/Library/.

[23] J. Esteves and J. Pastor. Enterprise resource planning systems research: An annotated bibliography. In Communications of AIS, 2001.

[24] ABB Automation Technology Products. IndustrialIT cpmPlus Enterprise Connectivity (version 4.0): Product Guide. ABB, 2009. 
[25] ISA-95 Group. Homepage. http://www.isa-95.com/.

[26] Zlib Project. Offical website. http://zlib.net.

[27] Libwww Project. Official website. http://www.w3.org/Library/.

[28] Libmodbus Project. Source website. http://copyleft.free.fr/ wordpress/index.php/libmodbus.

[29] ABB Automation Technology Products. IndustrialIT cpmPlus Enterprise Connectivity (version 4.0): Modeling Guidelines. ABB, 2009. 